\title{
Quantum stochastic convolution cocycles I
}

\author{
J. Martin Lindsay ${ }^{*}$, Adam G. Skalski ${ }^{1}$ \\ School of Mathematical Sciences, University of Nottingham, Nottingham, NG7 2RD, UK
}

Received 17 February 2004; accepted 19 October 2004

Available online 1 April 2005

Dedicated to the memory of Paul-André Meyer

\begin{abstract}
Stochastic convolution cocycles on a coalgebra are obtained by solving quantum stochastic differential equations. We describe a direct approach to solving such QSDE's by iterated quantum stochastic integration of matrix-sum kernels. The cocycles arising this way satisfy a Hölder condition, and it is shown that conversely every such Hölder-continuous cocycle is governed by a QSDE. Algebraic structure enjoyed by matrix-sum kernels yields a unital $*$-algebra of processes which allows easy deduction of homomorphic properties of cocycles on a 'quantum semigroup'. This yields a simple proof that every quantum Lévy process may be realised in Fock space. Finally perturbation of cocycles by Weyl cocycles is shown to be implemented by the action of the corresponding Euclidean group on Schürmann triples.
\end{abstract}

(c) 2005 Elsevier SAS. All rights reserved.

\section{Résumé}

Des cocycles de convolution stochastiques sur une coalgèbre sont obtenus par résolution des équations différentielles stochastiques (EDS) quantiques. Nous décrivons une méthode directe pour résoudre les EDS quantiques par intégration stochastique quantique itérée de noyaux matrice-somme. Les cocycles qui sont obtenus par cette méthode satisfont une condition de Hölder, et nous montrons réciproquement que chaque cocycle Hölder-continu est gouverné par une EDS quantique. La structure algébrique des noyaux matrice-somme donne une *-algèbre de processus, qui nous permet une déduction facile des propriétés homomorphiques de cocycles sur un groupe quantique. Ce résultat permet d'obtenir un argument simple pour montrer que chaque processus de Lévy quantique peut être realisé dans l'espace de Fock. Finalement, nous montrons que la perturbation des cocycles par des cocycles de Weyl est mise en oeuvre par l'action du groupe euclidien correspondant sur les triplets de Schürmann. (c) 2005 Elsevier SAS. All rights reserved.

Keywords: Noncommutative probability; Quantum group; Stochastic cocycle; Quantum Lévy process; Quantum stochastic

$M S C$ : primary $81 \mathrm{~S} 25 ;$ secondary $16 \mathrm{~W} 30$

\footnotetext{
* Corresponding author.

E-mail addresses: jml@maths.nott.ac.uk (J.M. Lindsay), adam.skalski@ maths.nottingham.ac.uk (A.G. Skalski).

1 Permanent address of AGS. Department of Mathematics, University of Łódź, ul. Banacha 22, 90-238 Łódź, Poland.
} 


\section{Introduction}

Stochastic cocycles on operator algebras [1] arise as solutions of noncommutative stochastic differential equations. In turn such cocycles may often be shown to have stochastic generators in the sense that they satisfy an exponential Itô type stochastic differential equation. In the context of quantum stochastic calculus [19,32,31,24] this has been studied in [18,20,2,8,10,3,27,29], and in an abstract context of noncommutative white noise in [16]. Quantum Lévy processes [5,34,35,13,36,31,12,11] are natural noncommutative analogues of stochastic semigroups [38]. They are defined with respect to a unital involutive bialgebra, in other words a quantum semigroup, and their construction was achieved by the use of integral-sum kernel operators on symmetric Fock space [30,31,23], extended to deal with a (possibly infinite-dimensional) multiplicity space for the noise $[35,36]$.

In this paper we analyse stochastic convolution cocycles on a coalgebra. The cocycle relation involves the coproduct

$$
k_{s+t}=k_{s} \star\left(\sigma_{s} \circ k_{t}\right),
$$

where $\alpha \star \beta:=\cdot(\alpha \otimes \beta) \mathbf{\Lambda}$, and the initial condition involves the counit

$$
k_{0}=\iota \circ \epsilon,
$$

$a \mapsto \epsilon(a) I$. The maps $\left(\sigma_{s}\right)_{s \geqslant 0}$ comprise a semigroup of shifts of the driving noise (a CCR flow in other parlance, see [4]), the (partially defined) product $\cdot$ multiplies independent parts of the noise algebra and $\iota$ is a simple ampliation.

Heeding Meyer's dictum: “The construction becomes very clear if we separate the coalgebraic and the algebraic structure" ([31], p. 204), we first establish existence and uniqueness for the coalgebra quantum stochastic differential equation

$$
\mathrm{d} k_{t}=\mathrm{d} \Lambda_{\varphi}(t) \star k_{t}
$$

with the same initial condition. Here $\varphi$ is any linear map from the coalgebra into $\mathcal{O}(\widehat{D})$, the linear space of operators on a Hilbert space $\hat{\mathrm{k}}:=\mathbb{C} \oplus \mathrm{k}$ with dense domain $\widehat{D}$. The solution acts on an exponential domain (with $D$-valued step functions as test functions), and is obtained as the composition of a linear map $v^{\varphi}$, from the coalgebra into a space of sequences in $\S_{D}:=\prod \mathcal{O}\left(\widehat{D}^{\otimes n}\right)$, and an 'integral': $k_{t}=\Lambda_{t} \circ v^{\varphi}$. Integration of a sequence is realised by summing the iterated quantum stochastic integrals of its terms (cf. [17,28]). Solutions form a cocycle in the above sense, with respect to the standard shift on Fock space over $L^{2}\left(\mathbb{R}_{+} ; \mathrm{k}\right)$, and are strongly $\frac{1}{2}$-Hölder-continuous. We show that conversely every such Hölder-continuous cocycle having a Hölder-continuous adjoint process satisfies a coalgebra quantum stochastic differential equation.

The integral $\Lambda$ is injective and is involutive, unital and (weakly) multiplicative, on suitable subspaces of $s_{D}$, for a simple matrix-sum convolution product on such sequences. This entails necessary and sufficient conditions on the generator $\varphi$ for the stochastic convolution cocycle to be unital, involutive or weakly multiplicative respectively, when the coalgebra is endowed with 'unit' or involution, or is a bialgebra. A priori the conditions involve the whole sequence of maps $v^{\varphi}$; we show how in fact they reduce to a condition on the first term only (cf. [28]). The paper ends with a discussion of the perturbation of stochastic convolution cocycles by operator cocycles (cf. [9,14]). Perturbation of quantum Lévy processes by 'Weyl' cocycles corresponds at the generator level precisely to the action of the Euclidean group on Schürmann triples (cf. [11]).

Schürmann's ingeneous original construction of quantum Lévy processes on Fock space [35,36], which has not hitherto been superseded, is somewhat complicated. As mentioned above the construction used families of threeargument integral-sum kernel operators [31]. We now see that augmenting the multiplicity space of the noise by one dimension and using single-argument block-matrix sum kernels both simplifies the construction considerably and reveals more clearly the algebraic structure. Indeed the kernels used here depend only on the cardinality of the (finite set) argument, and directly deliver processes so that there is no need to refer to a separately developed calculus of measurable families of kernels as in the original approach. Reconnecting with the spirit of Glockner's 
contribution [13], our approach is fully incorporated into the canon of quantum stochastic analysis [19,32,24]; it is thereby more easily adaptable for other uses and developments such as the study of completely positive $*-$ bialgebraic processes [37], and the construction and analysis of quantum Lévy processes on (locally) compact quantum groups $[40,41,22,21]$.

General notations. Set theoretic notations $\subset \subset$ and \# are used to denote respectively, subset of finite cardinality and, for such a set, its cardinality. In this paper tensor products are algebraic, unless adorned. Thus $\bar{\otimes}$ is used for the Hilbert space tensor product of spaces and bounded operators, whereas for unbounded operators $S$ and $T$, $S \otimes T$ denotes the operator with domain $\operatorname{Dom} S \otimes \operatorname{Dom} T$ and obvious action. Here $\operatorname{Dom} T$ denotes the domain of the operator $T$; similarly Ran $T$ denotes its image $\{T x: x \in \operatorname{Dom} T\}$. Tensor symbols between vectors are usually dropped when it is safe to do so. For a vector-valued function $f: \mathbb{R}_{+} \rightarrow V$ and subinterval $J$ of $\mathbb{R}_{+}, f_{J}$ denotes the function which coincides with $f$ on $J$ and vanishes outside $J$; this notation is also used for vectors $v$ by viewing them as constant functions. $L(V ; W)$ denotes the vector space of linear maps between vector spaces $V$ and $W$; $B(X ; Y)$ denotes the Banach space of bounded operators between Banach spaces $X$ and $Y$. Hilbert space inner products are linear in their second argument and we employ the following Dirac-inspired notation. For an element $\xi$ of a Hilbert space $\mathrm{h},|\xi\rangle \in B(\mathbb{C} ; \mathrm{h})$ and $\langle\xi| \in B(\mathrm{~h} ; \mathbb{C})$ are defined by

$$
|\xi\rangle: \alpha \mapsto \alpha \xi \quad \text { and } \quad\langle\xi|: \eta \mapsto\langle\xi, \eta\rangle
$$

we also write $E_{\xi}$ for $I_{\mathrm{k}} \bar{\otimes}|\xi\rangle$ or $|\xi\rangle \bar{\otimes} I_{\mathrm{k}}$ with context dictating which order and which Hilbert space k, and $E^{\xi}$ for $\left(E_{\xi}\right)^{*}$.

\section{Preliminaries}

Involution. For any map $\psi: S \rightarrow T$ between sets with involution, $\psi^{\dagger}: S \rightarrow T$ will denote the map $s \mapsto \psi\left(s^{*}\right)^{*}$. Thus if $\psi$ is a linear map between involutive vector spaces then so is $\psi^{\dagger}$, and if $\psi$ is an algebra homomorphism between $*$-algebras then so is $\psi^{\dagger}$; in all cases we call $\psi$ real if $\psi=\psi^{\dagger}$. Warning: when $\mathcal{A}_{1}$ and $\mathcal{A}_{2}$ are $*$-algebras, $L\left(\mathcal{A}_{1} ; \mathcal{A}_{2}\right)$ is a $*$-algebra under the pointwise product and involution ${ }^{\dagger}$; however when $\mathcal{A}_{2}=\mathcal{A}_{1}$ this is not the algebra one is usually interested in. For an involutive vector space $V,{ }^{\dagger}$ is a linear involution on the endomorphism algebra $L(V)$ but is homomorphic (rather than being antihomomorphic) and so is not an algebra involution in general. That said, it (obviously) becomes an algebraic involution once it is restricted to any Abelian subalgebra closed under $^{\dagger}$. This remark is relevant to present considerations since we are interested in one-parameter semigroups of maps.

The positive elements of an involutive algebra $\mathcal{A}$ are understood to be those that are expressible in the form $\sum_{i=1}^{n} a_{i}^{*} a_{i}$. Thus $\mathcal{A}_{+}$is a cone in $\mathcal{A}$ however, whereas $\mathcal{A}$ is the linear span of its hermitian elements, the inclusion Lin $\mathcal{A}_{+} \subset \mathcal{A}$ may be proper - in particular a positive map between involutive algebras $\psi: \mathcal{A}_{1} \rightarrow \mathcal{A}_{2}$ need not be real - when $\mathcal{A}_{1}$ is nonunital. A state on a unital $*$-algebra $\mathcal{A}$ is a positive linear functional $\omega: \mathcal{A} \rightarrow \mathbb{C}$ which is normalised, i.e. $\omega\left(1_{\mathcal{A}}\right)=1$.

Coalgebras and convolution semigroups. A complex vector space $\mathcal{C}$ is a coalgebra if there are linear maps $\boldsymbol{\Delta}: \mathcal{C} \rightarrow \mathcal{C} \otimes \mathcal{C}$ and $\epsilon: \mathcal{C} \rightarrow \mathbb{C}$, called the coproduct and counit respectively, enjoying coassociativity and the counit property, namely

$$
\begin{aligned}
& (\mathrm{id} \otimes \boldsymbol{\Delta}) \circ \boldsymbol{\Delta}=(\boldsymbol{\Delta} \otimes \mathrm{id}) \circ \boldsymbol{\Delta} \quad \text { and } \\
& (\mathrm{id} \otimes \epsilon) \circ \boldsymbol{\Delta}=(\epsilon \otimes \mathrm{id}) \circ \boldsymbol{\Lambda}=\mathrm{id}
\end{aligned}
$$


(regrettably the more usual symbol for coproduct is unavailable due to its ubiquitous use in quantum stochastics, and in this paper, as the orthogonal projection (2.1)). Sweedler has bequeathed the handy notation $a_{(1)} \otimes a_{(2)}$ for $\boldsymbol{\Delta} a$, in which both summation and indices are supressed [39]. With this, (1.1) and (1.2) read

$$
a_{(1)} \otimes a_{(2)(1)} \otimes a_{(2)(2)}=a_{(1)(1)} \otimes a_{(1)(2)} \otimes a_{(2)}, \quad \text { and } \quad a_{(1)} \epsilon\left(a_{(2)}\right)=\epsilon\left(a_{(1)}\right) a_{(2)}=a .
$$

Let $\boldsymbol{\Delta}_{0}:=$ id and for $n \in \mathbb{N}$ define

$$
\mathbf{\Delta}_{n}:=\left(\mathrm{id}^{\otimes(n-1)} \otimes \mathbf{\Lambda}\right) \circ \cdots \circ(\mathrm{id} \otimes \mathbf{\Delta}) \circ \mathbf{\Lambda},
$$

noting that coassociativity implies that moving any $\boldsymbol{\Delta}$ to any of the available tensor places within its bracket (rather than the right-most, as here) has no effect. It is easily verified that the family $\left\{\boldsymbol{\Lambda}_{n}: n \in \mathbb{Z}_{+}\right\}$enjoys the relationships

$$
\left(\boldsymbol{\Lambda}_{i} \otimes \mathbf{\Delta}_{j}\right) \circ \boldsymbol{\Lambda}=\boldsymbol{\Delta}_{i+j+1}
$$

The Sweedler notation extends to writing $a_{(1)} \otimes \cdots \otimes a_{(n+1)}$ for $\mathbf{\Delta}_{n} a(n \geqslant 1)$. Thus, for example, $a_{(1)} \otimes a_{(2)} \otimes a_{(3)}$ becomes a neutral notation for the effect of (1.1) on an element $a$.

The Fundamental Theorem on Coalgebras states that the coalgebra generated by a finite subset of a coalgebra is necessarily finite dimensional. This is an indispensible tool in the present context (see Lemma 4.3 below).

For linear maps $\alpha: \mathcal{C} \rightarrow U, \beta: \mathcal{C} \rightarrow V$ from a coalgebra into vector spaces, define

$$
\alpha \lesssim \beta:=(\alpha \otimes \beta) \circ \boldsymbol{\Delta}: \mathcal{C} \rightarrow U \otimes V .
$$

When there is a natural 'product' $U \otimes V \rightarrow W$ we write $\alpha \star \beta$ for the resulting map $\mathcal{C} \rightarrow W$. This notation will be useful in several contexts. Thus, for example, the counit property (1.2) implies that $\epsilon \star \alpha=\alpha \star \epsilon=\alpha$ for any linear map $\alpha$ from $\mathcal{C}$ into a vector space. In particular $(L(\mathcal{C} ; \mathbb{C}), \star)$ is a unital algebra with identity $\epsilon$.

A continuous convolution semigroup of functionals (CCSF, for short) on a coalgebra $\mathcal{C}$ :

$$
\kappa_{s+t}=\kappa_{s} \star \kappa_{t}, \quad \kappa_{t}(a) \rightarrow \epsilon(a) \quad \text { as } t \rightarrow 0 ;
$$

has a generator

$$
\gamma: a \mapsto \lim _{t \rightarrow 0} t^{-1}\left(\kappa_{t}(a)-\epsilon(a)\right)
$$

from which the semigroup may be recovered:

$$
\kappa_{t}=\exp _{\star} t \gamma: a \mapsto \sum_{n \geqslant 0}(n !)^{-1} t^{n} \gamma^{\star n}(a)
$$

where $\gamma^{\star 0}:=\epsilon$. The generator owes its existence to the Fundamental Theorem on Coalgebras and the following fact whose proof we include for the convenience of the reader; the convergence in (1.7) is similarly indebted.

Lemma 1.1. Let $\mathcal{C}$ be a coalgebra. The map $\kappa \mapsto \mathrm{id} \star \kappa=(\mathrm{id} \otimes \kappa) \circ \Delta$ defines an injective unital algebra homomorphism $R:(L(\mathcal{C} ; \mathbb{C}), \star) \rightarrow L(\mathcal{C})$, with left inverse $\phi \mapsto \epsilon \circ \phi$, which respects linear involution when $\mathcal{C}$ is involutive (see below). Moreover, the elements of $\operatorname{Ran} R$ leave each sub-coalgebra of $\mathcal{C}$ invariant.

Proof. In view of coassociativity and the identity

$$
\boldsymbol{\Delta} \circ(\mathrm{id} \otimes \kappa)=(\mathrm{id} \otimes \mathrm{id} \otimes \kappa) \circ(\boldsymbol{\Delta} \otimes \mathrm{id}),
$$

if $\kappa_{1}, \kappa_{2} \in L(\mathcal{C} ; \mathbb{C})$ then

$$
\begin{aligned}
\mathrm{id} \star\left(\kappa_{1} \star \kappa_{2}\right) & =\left\{\mathrm{id} \otimes\left(\left(\kappa_{1} \otimes \kappa_{2}\right) \circ \mathbf{\Delta}\right)\right\} \circ \mathbf{\Delta} \\
& =\left(\mathrm{id} \otimes \kappa_{1} \otimes \kappa_{2}\right) \circ(\mathrm{id} \otimes \mathbf{\Delta}) \circ \mathbf{\Delta} \\
& =\left(\mathrm{id} \otimes \kappa_{1}\right) \circ\left(\mathrm{id} \otimes \mathrm{id} \otimes \kappa_{2}\right) \circ(\mathbf{\Delta} \otimes \mathrm{id}) \circ \boldsymbol{\Delta} \\
& =\left(\mathrm{id} \otimes \kappa_{1}\right) \circ \mathbf{\Delta} \circ\left(\mathrm{id} \otimes \kappa_{2}\right) \circ \mathbf{\Delta},
\end{aligned}
$$


so $R$ is multiplicative. It is unital by the counit property. When $\mathcal{C}$ is involutive, $\boldsymbol{\Delta}$ is involutive so

$$
(\mathrm{id} \star \kappa)^{\dagger}(a)=\left((\operatorname{id} \otimes \kappa) \Delta a^{*}\right)^{*}=\left(\kappa\left(a_{(2)}^{*}\right) a_{(1)}^{*}\right)^{*}=\kappa^{\dagger}\left(a_{(2)}\right) a_{(1)}=\left(\operatorname{id} \otimes \kappa^{\dagger}\right)(\boldsymbol{\Delta} a),
$$

thus $R$ respects linear involution. By the counit property

$$
\epsilon \circ(\mathrm{id} \otimes \kappa) \circ \boldsymbol{\Delta}=\kappa \circ(\epsilon \otimes \mathrm{id}) \circ \boldsymbol{\Delta}=\kappa,
$$

so $R$ has left inverse $\phi \mapsto \epsilon \circ \phi$. The invariance is clear.

Further algebraic structure. An involution on a coalgebra $\mathcal{C}$ (mentioned in the previous lemma) is a vector space involution compatible with the coalgebra operations: $\epsilon\left(c^{*}\right)=\epsilon(c)^{*}, \boldsymbol{\Delta}\left(c^{*}\right)=\left(c_{(1)}\right)^{*} \otimes\left(c_{(2)}\right)^{*}$. A coalgebra is unital if it contains a specified element 1 satisfying $\epsilon(1)=1$ and $\mathbf{\Delta} 1=1 \otimes 1$. An algebra $\mathcal{A}$ is a bialgebra if it is also a coalgebra with multiplicative coproduct and counit. A $*$-bialgebra is a bialgebra with involution which is both algebraic and coalgebraic. Unitality for a bialgebra means that it is unital as an algebra and the coproduct and counit are unital.

Note the following traffic between properties of a CCSF $\left(\kappa_{t}\right)_{t} \geqslant 0$ and its generator $\gamma$ when the coalgebra has more structure. Each functional is real (respectively, unital) if and only if the generator is real (resp. vanishes at the unit). Moreover, on a $*$-bialgebra $\mathcal{B}$, if the functionals are positive then the generator is conditionally positive:

$$
\gamma(a) \geqslant 0 \text { for } a \in \mathcal{B}_{+} \cap \operatorname{Ker} \epsilon,
$$

since, for such elements $a$,

$$
t^{-1}\left(\kappa_{t}(a)-\epsilon(a)\right)=t^{-1} \kappa_{t}(a) \geqslant 0 \text { for all } t>0 .
$$

Spaces of unbounded operators. For a dense subspace $E$ of a Hilbert space $\mathrm{h}$ let $\mathcal{O}(E)$ denote the vector space of linear operators on $\mathrm{h}$ with domain $E$, and define subspaces as follows:

$$
\begin{aligned}
& \mathcal{O}^{\dagger}(E):=\left\{T \in \mathcal{O}(E) \mid \operatorname{Dom} T^{*} \supset E\right\}, \\
& \mathcal{O}^{\text {inv }}(E):=\{T \in \mathcal{O}(E) \mid \operatorname{Ran} T \subset E\} \text { and } \\
& \mathcal{O}^{*}(E):=\left\{T \in \mathcal{O}^{\dagger}(E) \mid T, T^{\dagger} \in \mathcal{O}^{\text {inv }}(E)\right\}
\end{aligned}
$$

(“inv" for invariant), where for $T \in \mathcal{O}^{\dagger}(E)$,

$$
T^{\dagger}:=\left.T^{*}\right|_{E} \text {. }
$$

Thus $\mathcal{O}^{\dagger}(E)$ is an involutive vector space, $\mathcal{O}^{\text {inv }}(E)$ is a unital algebra, and $\mathcal{O}^{*}(E)$ is a unital $*$-algebra; the former following from the inclusion $\operatorname{Dom}(S+\lambda T)^{*} \supset \operatorname{Dom} S^{*} \cap \operatorname{Dom} T^{*}$. To lighten notation in the sequel we write $I_{E}$, or simply $I$, for the identity element of these algebras, namely $\left.I\right|_{E}$. Clearly $\mathcal{O}(E) \cong L(E ; \mathrm{h})$ and $\mathcal{O}^{\text {inv }}(E) \cong L(E)$.

Operator composition $\mathcal{O}^{\dagger}(E) \times \mathcal{O}^{\text {inv }}(E) \rightarrow \mathcal{O}(E)$ extends to pairs $(S, T)$ in $\mathcal{O}^{\dagger}(E) \times \mathcal{O}(E)$ for which $\operatorname{Dom}\left(S^{\dagger}\right)^{*} \supset \operatorname{Ran} T$, as follows:

$$
S \cdot T:=\left(S^{\dagger}\right)^{*} T \text {. }
$$

This partially defined product is bilinear in an obvious sense. However, due to the vagaries of unbounded operators, associativity relations have to be justified. This said, we shall not need any of the well-developed theory [33] of algebras of unbounded operators here.

\section{Matrix-sum kernels}

In this section we identify some sequences of iterated quantum stochastic integrands, here dubbed matrixsum kernels, for solving quantum group quantum stochastic differential equations. The multiplicative structure of 
iterated quantum stochastic integrals is distilled in a simple convolution product on matrix-sum kernels involving only finite sums (see (2.2)). The convolution combines with bialgebra to establish multiplicative properties of solutions of quantum group QSDE's in Section 6.

Fix a dense subspace $D$ of a Hilbert space k, define

$$
\hat{\mathrm{k}}=\mathbb{C} \oplus \mathrm{k}, \quad \widehat{D}=\mathbb{C} \oplus D, \quad \text { and } \quad \Delta=P_{\mathrm{k}} \quad \text { (orthogonal projection) }
$$

and write $\widehat{D}^{\otimes}$ for the tensor algebra over $\widehat{D}$ viewed as a dense subspace of the full Fock space over $\hat{\mathrm{k}}$, with $\widehat{D}^{\otimes n}$ $(n \geqslant 0)$ viewed as subspaces. Also view $\mathcal{O}\left(\widehat{D}^{\otimes n}\right)$ as a subspace of $\mathcal{O}\left(\widehat{D}^{\otimes}\right)$. Let $\wp_{D}$ denote the vector space of maps $F: \mathbb{Z}_{+} \rightarrow \mathcal{O}\left(\widehat{D}^{\otimes}\right)$ such that $F_{n} \in \mathcal{O}\left(\widehat{D}^{\otimes n}\right)$ and consider the following subspaces of $s_{D}$, in which $F^{\dagger}$ is defined pointwise: $F_{n}^{\dagger}:=\left(F_{n}\right)^{\dagger}$, when $F$ is $\mathcal{O}^{\dagger}\left(\widehat{D}^{\otimes}\right)$-valued:

$$
\begin{aligned}
& s_{D}^{\dagger}:=\left\{F \in s_{D} \mid \forall_{n \in \mathbb{Z}_{+}} F_{n} \in \mathcal{O}^{\dagger}\left(\widehat{D}^{\otimes n}\right)\right\}, \\
& s_{D}^{\text {inv }}:=\left\{F \in s_{D} \mid \forall_{n \in \mathbb{Z}_{+}} F_{n} \in \mathcal{O}^{\text {inv }}\left(\widehat{D}^{\otimes n}\right)\right\} \quad \text { and } \\
& s_{D}^{*}:=\left\{F \in \wp_{D}^{\dagger} \mid F, F^{\dagger} \in s_{D}^{\text {inv }}\right\} .
\end{aligned}
$$

Clearly $\wp_{D}^{\dagger}$ is an involutive vector space. Next consider the matrix-sum convolution product $*: \wp_{D} \times \wp_{D}^{\text {inv }} \rightarrow \varsigma_{D}$, given by

$$
(F * G)_{n}=\sum_{|\alpha|=\{1, \ldots, n\}} F\left(\alpha_{1} \cup \alpha_{2} ; n\right) \Delta\left[\alpha_{2} ; n\right] G\left(\alpha_{2} \cup \alpha_{3} ; n\right)
$$

where the sum is over all $3^{n}$ disjoint partitions $\alpha_{1} \cup \alpha_{2} \cup \alpha_{3}$ of $\{1, \ldots, n\}$, and the components of the summands are defined as follows: for $F \in S_{D}$ and $\alpha \subset\{1, \ldots, n\}$ define

$$
F(\alpha ; n):=\Pi_{\alpha ; n}^{*}\left(F_{k} \otimes I_{n-k}\right) \Pi_{\alpha ; n} \in \mathcal{O}\left(\widehat{D}^{\otimes n}\right)
$$

where, writing $\alpha=\left\{\alpha_{1}<\cdots<\alpha_{k}\right\}$ and $\{1, \ldots, n\} \backslash \alpha=\left\{\bar{\alpha}_{1}<\cdots<\bar{\alpha}_{n-k}\right\}, \Pi_{\alpha ; n} \in \mathcal{O}^{*}\left(\widehat{D}^{\otimes n}\right)$ is the linear extension of the map

$$
\chi_{1} \otimes \cdots \otimes \chi_{n} \mapsto \chi_{\alpha_{1}} \otimes \cdots \otimes \chi_{\alpha_{k}} \otimes \chi_{\bar{\alpha}_{1}} \otimes \cdots \otimes \chi_{\bar{\alpha}_{n-k}},
$$

and, (with $\Delta$ defined in (2.1))

$$
\Delta[\alpha ; n]:=\Delta^{\otimes}(\alpha ; n) \quad \text { where } \Delta_{n}^{\otimes}:=\Delta^{\otimes n} .
$$

Thus if $F_{n}$ is a simple tensor $T_{1} \otimes \cdots \otimes T_{n}$, as is the case for $F=\Delta^{\otimes}$, then $F(\alpha ; n)=S_{1} \otimes \cdots \otimes S_{n}$ where

$$
S_{i}= \begin{cases}T_{i} & \text { if } i \in \alpha \\ I & \text { otherwise. }\end{cases}
$$

This is the product which reflects multiplication of iterated quantum stochastic integrals, as we shall see.

Lemma 2.1. The product enjoys the following properties:

(a) If $F, G \in 8_{D}^{\text {inv }}$ then $F * G \in 8_{D}^{\text {inv }}$;

(b) If $F \in \varsigma_{D}$ and $G, H \in s_{D}^{\text {inv }}$ then $F *(G * H)=(F * G) * H$;

(c) If $E=1 \delta_{0}$ then $E \in s_{D}^{*}$ and $E * F=F * E=F$ for all $F \in s_{D}$;

(d) If $F, G \in \mathcal{S}_{D}^{\dagger}$ with $G, F^{\dagger} \in \mathcal{S}_{D}^{\text {inv }}$ then $F * G \in \mathcal{S}_{D}^{\dagger}$ and $(F * G)^{\dagger}=G^{\dagger} * F^{\dagger}$.

In particular, $\left(\delta_{D}^{*}, *\right)$ is a unital $*$-algebra. 
Proof. To see (b) note that

$$
\sum_{|\boldsymbol{\alpha}|=\{1, \ldots, n\}} F\left(\alpha_{1} \cup \alpha_{2} ; n\right) \Delta\left[\alpha_{2} ; n\right] G\left(\alpha_{2} \cup \alpha_{3} ; n\right) \Delta\left[\alpha_{3} ; n\right] H\left(\alpha_{3} \cup \alpha_{4} ; n\right)
$$

is a common expression for $(F *(G * H))_{n}$ and $((F * G) * H)_{n}$. The rest is easily verified.

For quantum stochastic purposes we need to impose a growth condition. Thus let $\mathcal{G}_{D}$ denote those $F \in \varsigma_{D}$ satisfying

$$
\forall_{S \subset \subset \widehat{D}} \exists_{C_{1}, C_{2}>0} \forall_{n \in \mathbb{Z}_{+}, \chi_{1}, \ldots, \chi_{n} \in S} \quad\left\|F_{n}\left(\chi_{1} \otimes \cdots \otimes \chi_{n}\right)\right\| \leqslant C_{1} C_{2}^{n},
$$

and define subspaces

$$
\begin{aligned}
& \mathcal{G}_{D}^{\dagger}:=\left\{F \in \mathcal{S}_{D}^{\dagger} \mid F, F^{\dagger} \in \mathcal{G}_{D}\right\}, \\
& \mathcal{G}_{D}^{\text {inv }}:=\mathcal{G}_{D} \cap \mathcal{S}_{D}^{\text {inv }} \text { and } \\
& \mathcal{G}_{D}^{*}:=\left\{F \in \mathcal{G}_{D}^{\dagger} \mid F, F^{\dagger} \in \mathcal{G}_{D}^{\text {inv }}\right\} .
\end{aligned}
$$

To obtain algebras of processes we need to restrict further. Our choice of restriction here is manifestly informed by the Fundamental Theorem of Coalgebra. Thus let $\mathcal{H}_{D}$ denote the set of $F \in \varsigma_{D}$ satisfying

$\exists_{p, q \in \mathbb{N}, R \subset \subset \mathcal{O}(\widehat{D})} \forall_{n \in \mathbb{Z}_{+}} F_{n}$ may be expressed as a sum of $p q^{n}$ terms

of the form $X_{1} \otimes \cdots \otimes X_{n}$ with $X_{1}, \ldots, X_{n} \in R$,

with $\mathcal{H}_{D}^{\dagger}, \mathcal{H}_{D}^{\text {inv }}$ and $\mathcal{H}_{D}^{*}$ defined as for $\mathcal{G}$. All of these are subspaces of $\mathcal{G}_{D}$.

Proposition 2.2. Let $F \in \mathcal{G}_{D}$ and $G \in \mathcal{H}_{D}^{\text {inv }}$. Then $F * G \in \mathcal{G}_{D}$, moreover if $F \in \mathcal{H}_{D}$ then $F * G \in \mathcal{H}_{D}$ too.

Proof. Let $H=F * G$ and choose $p, q$ and $R$ for $G$ according to (2.6). Let $S \subset \subset \widehat{D}$ and let $n \in \mathbb{N}$ and $\chi_{1}, \ldots \chi_{n} \in S$. Then, for any partition $\alpha \cup \beta \cup \gamma$ of $\{1, \ldots, n\}$,

$$
F(\alpha \cup \beta ; n) \Delta[\beta ; n] G(\beta \cup \gamma ; n)\left(\chi_{1} \otimes \cdots \otimes \chi_{n}\right)
$$

is a sum of $p q^{\#(\beta \cup \gamma)}$ terms of the form

$$
F(\alpha \cup \beta ; n)\left(\eta_{1} \otimes \cdots \otimes \eta_{n}\right)
$$

where each $\eta_{i}$ belongs to the finite set $S^{\prime}:=R S \cup \Delta R S$. Thus, choosing $C_{1}$ and $C_{2} \geqslant 1$ for the pair $\left(F, S^{\prime}\right)$ according to (2.4), and setting $M=\max \left\{\|\eta\|: \eta \in S^{\prime}\right\}$,

$$
\left\|H_{n}\left(\chi_{1} \otimes \cdots \otimes \chi_{n}\right)\right\| \leqslant \sum p q^{\#(\beta \cup \gamma)} C_{1} C_{2}^{\#(\alpha \cup \beta)} M^{\# \gamma}=C_{1}^{\prime}\left(C_{2}^{\prime}\right)^{n},
$$

where $C_{1}^{\prime}=p C_{1}$ and $C_{2}^{\prime}=\left(C_{2}+q C_{2}+q M\right)$. Thus $H \in \mathcal{G}_{D}$.

If $F \in \mathcal{H}_{D}$ then, choosing $p^{\prime}, q^{\prime}$ and $R^{\prime}$ for $F$ (and assuming without loss that $\left.I \in R \cap R^{\prime}\right), F(\alpha \cup$ $\beta ; n) \Delta[\beta ; n] G(\beta \cup \gamma ; n)$ is a sum of $p^{\prime}\left(q^{\prime}\right)^{\#(\alpha \cup \beta)} p q^{\#(\beta \cup \gamma)}$ terms of the form $Z_{1} \otimes \cdots \otimes Z_{n}$ where $Z_{i} \in$ $R^{\prime} R \cup R^{\prime} \Delta R$. Thus $H_{n}$ is a sum of $p p^{\prime}\left(q+q q^{\prime}+q^{\prime}\right)^{n}$ terms of this form. Thus $H \in \mathcal{H}_{D}$.

Remark. There are alternative hypotheses which are also useful, for example: $F \in \mathcal{G}_{D}^{\dagger}, G \in \mathcal{H}_{D}$ and the finite set $R$ may be chosen so that $X_{1} \chi_{1} \otimes \cdots \otimes X_{k} \chi_{k} \in \operatorname{Dom} F_{k}^{\dagger *}$, for all $k \in \mathbb{N}, X_{1}, \ldots, X_{k} \in R$ and $\chi_{1}, \ldots, \chi_{k} \in \widehat{D}$. This is relevant to remarks in Section 6.

As an immediate consequence we have the following result.

Theorem 2.3. $\left(\mathcal{H}_{D}^{*}, *\right)$ is a unital $*$-subalgebra of $\left(\mathcal{(}_{D}^{*}, *\right)$. 
This algebra is sufficiently regular to admit quantum stochastic integration (Theorem 3.3 below) whilst being sufficiently large to house the mechanism for solving coalgebra QSDE's (Theorem 4.4).

\section{Quantum stochastics}

Fock space. For a subinterval $J$ of $\mathbb{R}_{+}$and Hilbert space $\mathrm{k}, \mathcal{F}_{\mathrm{k}, J}$ denotes the symmetric Fock space over $L^{2}(J ; \mathrm{k})$ with $\mathcal{F}_{\mathrm{k}}:=\mathcal{F}_{\mathrm{k}, \mathbb{R}_{+}}$. By the exponential property $\mathcal{F}_{\mathrm{k},[a, b[} \bar{\otimes} \mathcal{F}_{\mathrm{k},[b, c[}$ is identified as $\mathcal{F}_{\mathrm{k},[a, c[}$ for $0 \leqslant a \leqslant b \leqslant c \leqslant \infty$. Exponential vectors are denoted $\varepsilon(f)$ for $f \in L^{2}(J ; \mathrm{k})$ with $\varepsilon(0)$ denoted $\Omega_{\mathrm{k}, J}$. For $t \geqslant 0, \gamma_{t}^{\mathrm{k}}$ denotes the shift $B\left(\mathcal{F}_{\mathrm{k}}\right) \rightarrow B\left(\mathcal{F}_{\mathrm{k},[t, \infty[}\right)$ so that the $C C R$ flow of index $\mathrm{k}$ is given by

$$
\sigma_{t}^{\mathrm{k}}: B\left(\mathcal{F}_{\mathrm{k}}\right) \rightarrow B\left(\mathcal{F}_{\mathrm{k}}\right), \quad T \mapsto I_{\mathrm{k},[0, t[} \bar{\otimes} \gamma_{t}^{\mathrm{k}}(T),
$$

where $I_{\mathrm{k}, J}$ is used to denote the identity on $\mathcal{F}_{\mathrm{k}, J}$. Since $\gamma_{t}^{\mathrm{k}}$ is implemented by a unitary operator $\mathcal{F}_{\mathrm{k}} \rightarrow \mathcal{F}_{\mathrm{k},[t, \infty[}$, these extend to maps of unbounded operators $\mathcal{O}\left(\mathcal{E}_{D}\right) \rightarrow \mathcal{O}\left(\mathcal{E}_{D,[t, \infty[}\right)\left(\right.$ resp. $\left.\mathcal{O}\left(\mathcal{E}_{D}\right)\right)$ where, for a subset $D$ of $\mathrm{k}$,

$$
\mathcal{E}_{D, J}:=\operatorname{Lin}\left\{\varepsilon(f): f \in \mathbb{S}_{D, J}\right\}
$$

and $\mathbb{S}_{D, J}:=\operatorname{Lin}\left\{d_{[a, b[}: d \in D,\left[a, b[\subset J\}\right.\right.$; we set $\mathcal{E}_{D}:=\mathcal{E}_{D, \mathbb{R}_{+}}$. Similarly the vector state $\omega_{\Omega}$ on $B\left(\mathcal{F}_{\mathrm{k}}\right.$ ) (where $\left.\Omega:=\Omega_{\mathrm{k}, \mathbb{R}_{+}}\right)$extends to $\mathcal{O}\left(\mathcal{E}_{D}\right)$ by the same formula. Once $\mathrm{k}$ or $D$ is fixed then it is dropped from the notation.

Convention. We do not notationally distinguish between an operator $X_{s}$ of the form $X \otimes I_{[s, \infty[} \mid \mathcal{E}_{D}$ and the operator $X \otimes I_{[s, \infty[}$. In this way $X_{s} \sigma_{s}(T)$ makes sense for $T \in \mathcal{O}\left(\mathcal{E}_{D}\right)$; moreover, by further minor abuse we sometimes denote it

$$
X_{S} \otimes \gamma_{s}(T)
$$

This is consistent with the partially defined product (1.10).

Processes and integrals. Fix now, once and for all, a dense subspace $D$ of a Hilbert space k. Let $\mathcal{E}_{D}$ denote the exponential domain $\operatorname{Lin}\left\{\varepsilon(f) \mid f \in \mathbb{S}_{D}\right\}$ where $\mathbb{S}_{D}=\operatorname{Lin}\left\{d_{[0, t[} \mid d \in D, t>0\right\}$. Following the notation and terminology adopted in [28], for $\mathcal{D}$ of the form $E \otimes \mathcal{E}_{D}$, where $E$ is a dense subspace of a Hilbert space h, let $\mathbb{P}(\mathcal{D})$ denote the vector space of (adapted, weakly measurable) operator h-processes with domain $\mathcal{D}$, with its subspaces

$$
\begin{aligned}
& \mathbb{P}_{\mathrm{Hc}}(\mathcal{D}):=\left\{X \in \mathbb{P}(\mathcal{D}): \forall_{\xi \in \mathcal{D}} t \mapsto X_{t} \xi \text { is locally Hölder-continuous with exponent } \frac{1}{2}\right\}, \\
& \mathbb{P}_{\mathrm{c}}(\mathcal{D}):=\left\{X \in \mathbb{P}(\mathcal{D}): \forall_{\xi \in \mathcal{D}} t \mapsto X_{t} \xi \text { is continuous }\right\}, \\
& \mathbb{P}_{\mathrm{lb}}(\mathcal{D}):=\left\{X \in \mathbb{P}(\mathcal{D}): \forall_{\xi \in \mathcal{D}} t \mapsto X_{t} \xi \text { is (measurable and) locally bounded }\right\}, \\
& \mathbb{P}_{2}(\mathcal{D}):=\left\{X \in \mathbb{P}(\mathcal{D}): \forall_{\xi \in \mathcal{D}} t \mapsto X_{t} \xi \text { is locally square-integrable }\right\}, \\
& \mathbb{P}_{\mathrm{wc}}(\mathcal{D}):=\left\{X \in \mathbb{P}(\mathcal{D}): \forall_{\xi, \xi^{\prime} \in \mathcal{D}} t \mapsto\left\langle\xi, X_{t} \xi^{\prime}\right\rangle \text { is continuous }\right\}, \\
& \mathbb{P}_{\mathrm{wr}}(\mathcal{D}):=\left\{X \in \mathbb{P}(\mathcal{D}): \forall_{f, g \in \mathbb{S}_{D}} E^{\varepsilon(f)} X_{t} E_{\varepsilon(g)} \text { is bounded, locally uniformly in } t\right\}
\end{aligned}
$$

of (Hölder-)continuous, locally bounded, square-integrable, weakly continuous and weakly regular processes respectively. Integrability here is in the Bochner sense and equality of processes $X$ and $Y$ means that, for each $\xi \in \mathcal{D}$, $X_{t} \xi=Y_{t} \xi$ for almost all $t$. In the definition of $\mathbb{P}_{\text {wr }}$ the notation described below $(0.1)$ is used. If $E=\mathbb{C}$ then $\mathbb{P}_{\mathrm{wc}}(\mathcal{D}) \subset \mathbb{P}_{\mathrm{wr}}(\mathcal{D})$. Also let

$$
\begin{aligned}
& \mathbb{P}^{\dagger}(\mathcal{D}):=\left\{X \in \mathbb{P}(\mathcal{D}) \mid \forall_{t \geqslant 0} X_{t} \in \mathcal{O}^{\dagger}(\mathcal{D})\right\} \quad \text { and } \\
& \mathbb{P}_{\alpha}^{\dagger}(\mathcal{D}):=\left\{X \in \mathbb{P}^{\dagger}(\mathcal{D}) \mid X, X^{\dagger} \in \mathbb{P}_{\alpha}(\mathcal{D})\right\},
\end{aligned}
$$

where $\alpha$ may be any of the above subscripts and $X \mapsto X^{\dagger}$ is the pointwise induced linear involution on $\mathbb{P}^{\dagger}(\mathcal{D})$.

Quantum stochastic integration [32,24] gives a linear map

$$
\mathbb{P}_{2}\left(E \otimes \widehat{D} \otimes \mathcal{E}_{D}\right) \rightarrow \mathbb{P}_{\mathrm{c}}\left(E \otimes \mathcal{E}_{D}\right),
$$


denoted $\int_{0}^{\cdot} L(s) \mathrm{d} \Lambda(s)$, enjoying the Fundamental Formulae and Fundamental Estimate below. Let $x, y \in E, f, g \in$ $\mathbb{S}_{D}$ and $0 \leqslant r \leqslant t \leqslant T$, and let $X=\int_{0}^{\cdot} L(s) \mathrm{d} \Lambda(s)$ and $Y=\int_{0}^{\cdot} M(s) \mathrm{d} \Lambda(s)$. Then

$$
\begin{aligned}
& \langle x \varepsilon(f), X(t) y \varepsilon(g)\rangle=\int_{0}^{t}\langle\zeta(s), L(s) \eta(s)\rangle \mathrm{d} s, \\
& \begin{aligned}
\langle X(t) x \varepsilon(f), X(t) y \varepsilon(g)\rangle= & \int_{0}^{t}\{\langle L(s) \zeta(s), \tilde{Y}(s) \eta(s)\rangle+\langle\tilde{X}(s) \zeta(s), M(s) \eta(s)\rangle \\
& +\langle L(s) \zeta(s), \Delta M(s) \eta(s)\rangle\} \mathrm{d} s \quad \text { and }
\end{aligned} \\
& \begin{array}{l}
\|[X(t)-X(r)] x \varepsilon(f)\|^{2} \leqslant C(f, T) \int_{r}^{t}\|L(s) \zeta(s)\|^{2} \mathrm{~d} s,
\end{array}
\end{aligned}
$$

where $\zeta(s):=x \hat{f}(s) \varepsilon(f), \eta(s):=y \hat{g}(s) \varepsilon(g)$ and $C$ is a constant depending only on $f$ and $T$. The tilde notation here is defined by $\widetilde{X}(s)=\tau\left(I_{\hat{\mathrm{k}}} \otimes X(s)\right)$ where $\tau$ is the tensor flip $\mathcal{O}\left(\widehat{D} \otimes E \otimes \mathcal{E}_{D}\right) \rightarrow \mathcal{O}\left(E \otimes \widehat{D} \otimes \mathcal{E}_{D}\right)$. Moreover, if $L \in \mathbb{P}_{2}^{\dagger}\left(E \otimes \widehat{D} \otimes \mathcal{E}_{D}\right)$ then $X \in \mathbb{P}_{c}^{\dagger}\left(E \otimes \mathcal{E}_{D}\right)$ and

$$
\left(\int_{0}^{t} L(s) \mathrm{d} \Lambda(s)\right)^{\dagger}=\int_{0}^{t} L^{\dagger}(s) \mathrm{d} \Lambda(s) .
$$

Quantum stochastic integration is injective ([26], Proposition 2.2). Moreover, from (3.6) it is clear that (3.5) restricts to a map

$$
\mathbb{P}_{\mathrm{lb}}\left(E \otimes \widehat{D} \otimes \mathcal{E}_{D}\right) \rightarrow \mathbb{P}_{\mathrm{Hc}}\left(E \otimes \mathcal{E}_{D}\right)
$$

Iterated quantum stochastic integrals. For $L \in \mathcal{O}\left(E \otimes \widehat{D}^{\otimes n}\right)$, where $E$ is a dense subspace of a Hilbert space h, define $\Lambda^{n}(L) \in \mathbb{P}_{\mathrm{c}}\left(E \otimes \mathcal{E}_{D}\right)$ recursively as follows:

$$
\Lambda_{t}^{0}(L)=\left.L \otimes I\right|_{\mathcal{E}_{D}}, \quad \text { and, } \quad \text { for } n \geqslant 1, \quad \Lambda_{t}^{n}(L)=\int_{0}^{t} \Lambda_{s}^{n-1}(L) \mathrm{d} \Lambda(s),
$$

by viewing $E \otimes \widehat{D}^{\otimes n}$ as $(E \otimes \widehat{D}) \otimes \widehat{D}^{\otimes(n-1)}$. Letting $x, y, f, g$ and $T$ be as in the Fundamental Formulae above, these satisfy the identity

$$
\left\langle x \varepsilon(f), \Lambda_{t}^{n}(L) y \varepsilon(g)\right\rangle=\int_{\Delta_{n}[0, t]}\left\langle\zeta(s),\left(L \otimes I_{\mathcal{F}}\right) \eta(s)\right\rangle \mathrm{d} s,
$$

and estimate

$$
\left\|\Lambda_{t}^{n}(L) x \varepsilon(f)\right\|^{2} \leqslant C(f, T)^{n} \int_{\Delta_{n}[0, t]}\left\|\left(L \otimes I_{\mathcal{F}}\right) \zeta(s)\right\|^{2} \mathrm{~d} \boldsymbol{s},
$$

where $\Delta_{n}[0, t]$ is the simplex $\left\{s \in[0, t]^{n} \mid s_{n} \geqslant \cdots \geqslant s_{1}\right\}$, and we are using the notation $\zeta(s):=x \hat{f}^{\otimes n}(s) \varepsilon(f)$ where $\hat{f}^{\otimes n}(s):=\hat{f}\left(s_{n}\right) \cdots \hat{f}\left(s_{1}\right)$ and similarly for $\eta$. Moreover if $L \in \mathcal{O}^{\dagger}\left(E \otimes \widehat{D}^{\otimes n}\right)$ then $\Lambda^{n}(L) \in \mathbb{P}_{\mathrm{c}}^{\dagger}\left(E \otimes \mathcal{E}_{D}\right)$ and

$$
\Lambda_{t}^{n}\left(L^{\dagger}\right)=\Lambda_{t}^{n}(L)^{\dagger}
$$


The multiplicative structure of iterated integrals is revealed by embracing a sequence of them at once. Note immediately that if $F \in \mathcal{G}_{D}$ then $\sum_{n \geqslant 0} \Lambda_{t}^{n}\left(F_{n}\right) \varepsilon(f)$ converges absolutely, and the convergence is locally uniform in $t$. Clearly the resulting map

$$
\Lambda: \mathcal{G}_{D} \rightarrow \mathbb{P}_{\mathrm{c}}\left(\mathcal{E}_{D}\right)
$$

is linear. In the Guichardet notation $\Gamma_{J}:=\{\sigma \subset J \mid \# \sigma<\infty\}$ for $J \subset \mathbb{R}_{+}$,

$$
\pi_{\hat{f}}(\sigma)=\hat{f}^{\otimes n}(s) \quad \text { for } \sigma=\left\{s_{n}>\cdots>s_{1}\right\}
$$

(3.9) yields the useful identity

$$
\left\langle\varepsilon(f), \Lambda_{t}(F) \varepsilon(g)\right\rangle=\int_{\Gamma_{[0, t]}} \mathrm{d} \sigma\left\langle\pi_{\hat{f}}(\sigma), F_{\# \sigma} \pi_{\hat{g}}(\sigma)\right\rangle\langle\varepsilon(f), \varepsilon(g)\rangle,
$$

where $\int \mathrm{d} \sigma$ denotes integration with respect to the symmetric measure of Lebesgue measure [15].

Using the Fundamental Estimates for (iterated) quantum stochastic integration it is easily verified that

$$
\Lambda\left(\mathcal{G}_{D}\right) \subset \mathbb{P}_{\mathrm{Hc}}\left(\mathcal{E}_{D}\right) .
$$

If $F \in \mathcal{G}_{D}^{\dagger}$ then it follows from (3.11) that $\Lambda(F) \in \mathbb{P}_{\mathrm{Hc}}^{\dagger}\left(\mathcal{E}_{D}\right)$, and

$$
\Lambda\left(F^{\dagger}\right)=\Lambda(F)^{\dagger} \text {. }
$$

The argument given in the proof of Proposition 2.3 of [28] yields injectivity of the map $\Lambda$, whereas those of Theorem 2.2 of that paper yield the following.

Theorem 3.1. Let $F \in \mathcal{G}_{D}^{\dagger}$ and $G \in \mathcal{G}_{D}^{\text {inv }}$. Then, for each $N \in \mathbb{Z}_{+}$,

$$
\sum_{i, j=0}^{N}\left\langle\Lambda_{t}^{i}\left(F_{i}\right) \varepsilon(f), \Lambda_{t}^{j}\left(G_{j}\right) \varepsilon(g)\right\rangle=\sum_{k=0}^{2 N}\left\langle\varepsilon(f), \Lambda_{t}^{k}\left(H_{k}\right) \varepsilon(g)\right\rangle
$$

where $H=F^{\dagger} * G$.

Recall the partially defined product (1.10).

Proposition 3.2. Let $F \in \mathcal{G}_{D}^{\dagger}$ and $G \in \mathcal{G}_{D}^{\text {inv }}$ be such that $F * G \in \mathcal{G}_{D}$. Then, for each $t \geqslant 0$, Dom $\Lambda_{t}(F)^{\dagger *} \supset$ $\operatorname{Ran} \Lambda_{t}(G)$ and

$$
\Lambda_{t}(F * G)=\Lambda_{t}(F) \cdot \Lambda_{t}(G) .
$$

Proof. For $H \in \S_{D}$ and $N \geqslant 0$ write $\Lambda^{[N]}(H)$ for $\sum_{i=0}^{N} \Lambda^{i}\left(H_{i}\right) \in \mathbb{P}_{\mathrm{c}}\left(\mathcal{E}_{D}\right)$, so that if $H \in \mathcal{G}_{D}$ then $\Lambda_{t}^{[N]}(H) \varepsilon(f) \rightarrow$ $\Lambda_{t}(H) \varepsilon(f)$ as $N \rightarrow \infty$. By Theorem 3.1 and (3.11)

$$
\left\langle\Lambda_{t}(F)^{\dagger} \varepsilon(f), \Lambda_{t}(G) \varepsilon(g)\right\rangle=\lim _{N \rightarrow \infty}\left\langle\varepsilon(f), \Lambda_{t}^{[N]}(F * G) \varepsilon(g)\right\rangle=\left\langle\varepsilon(f), \Lambda_{t}(F * G) \varepsilon(g)\right\rangle,
$$

and so the result follows.

The following is an immediate consequence of this and Theorem 2.3.

Theorem 3.3. Let $\mathcal{P}=\Lambda\left(\mathcal{H}_{D}^{*}\right) \subset \mathbb{P}_{\mathrm{Hc}}^{\dagger}\left(\mathcal{E}_{D}\right)$. Then, with respect to the product defined in $(1.10)$ - extended pointwise, the map $\Lambda$ restricts to a unital $*$-algebra isomorphism of unital $*$-algebras:

$$
\left(\mathcal{H}_{D}^{*}, *\right) \rightarrow(\mathcal{P}, \cdot)
$$




\section{Coalgebra stochastic differential equations}

For this section and the next we fix a coalgebra $\mathcal{C}$.

Coalgebraic processes. A linear map $k: \mathcal{C} \rightarrow \mathbb{P}(\mathcal{D})$, where $\mathcal{D}$ is of the form $E \otimes \mathcal{E}_{D}$ for a dense subspace $E$ of a Hilbert space $\mathrm{h}$, is called an $\mathrm{h}$-process on $\mathcal{C}$ with domain $\mathcal{D}$ (dropping the $\mathrm{h}$ - when $\mathrm{h}=\mathbb{C}$ ). The vector space consisting of these is denoted $\mathbb{P}(\mathcal{C}, \mathcal{D})$, with subspaces

$$
\begin{aligned}
& \mathbb{P}_{\alpha}(\mathcal{C}, \mathcal{D}):=\left\{k \in \mathbb{P}(\mathcal{C}, \mathcal{D}) \mid k(\mathcal{C}) \subset \mathbb{P}_{\alpha}(\mathcal{D})\right\}, \\
& \mathbb{P}_{\alpha}^{\dagger}(\mathcal{C}, \mathcal{D}):=\left\{k \in \mathbb{P}(\mathcal{C}, \mathcal{D}) \mid k(\mathcal{C}) \subset \mathbb{P}_{\alpha}^{\dagger}(\mathcal{D})\right\}
\end{aligned}
$$

where $\alpha$ is any of the available subscripts from (3.4). Usually for us $\mathcal{D}$ will simply be $\mathcal{E}_{D}$. An involution on $\mathcal{C}$ induces an involution on $\mathbb{P}^{\dagger}(\mathcal{C}, \mathcal{D}): k^{\dagger}(x):=k .\left(x^{*}\right)^{\dagger}$. A process $k$ in $\mathbb{P}^{\dagger}\left(\mathcal{C}, \mathcal{E}_{D}\right)$ is then called real if $k^{\dagger}=k$. If $\mathcal{C}$ is unital, respectively a bialgebra, then $k$ is unital if $k_{t}(1)=I$ for all $t \geqslant 0$, resp. (weakly) multiplicative if

$$
\operatorname{Dom} k_{t}(a)^{\dagger *} \supset \operatorname{Ran} k_{t}(b) \text { and } k_{t}(a) \cdot k_{t}(b)=k_{t}(a b)
$$

for all $a, b \in \mathcal{C}$ and $t \geqslant 0$.

Remark. Note the following inclusion

$$
\mathbb{P}_{\text {wc }}\left(\mathcal{C}, \mathcal{E}_{D}\right) \subset \mathbb{P}_{\text {wr }}\left(\mathcal{C}, \mathcal{E}_{D}\right),
$$

which follows from the corresponding inclusion for operator processes: $\mathbb{P}_{\mathrm{wc}}\left(\mathcal{E}_{D}\right) \subset \mathbb{P}_{\mathrm{wr}}\left(\mathcal{E}_{D}\right)$.

Lemma 4.1. Let $k \in \mathbb{P}_{\mathrm{wr}}\left(\mathcal{C}, \mathcal{E}_{D}\right)$ and let $V$ be a finite dimensional subspace of $\mathcal{C}$ equipped with some norm. Then, for each $f, g \in \mathbb{S}_{D}$ and $T \geqslant 0$,

$$
C_{f, g, T, V}:=\sup \left\{\left|\left\langle\varepsilon(f), k_{t}(x) \varepsilon(g)\right\rangle\right|: x \in V,\|x\| \leqslant 1,0 \leqslant t \leqslant T\right\}<\infty .
$$

Proof. Let $e_{1}, \ldots e_{N}$ be a basis for $V$. Then, for $x \in V$,

$$
\left|\left\langle\varepsilon(f), k_{t}(x) \varepsilon(g)\right\rangle\right| \leqslant\|x\|^{\prime} \max _{i}\left|\left\langle\varepsilon(f), k_{t}\left(e_{i}\right) \varepsilon(g)\right\rangle\right|
$$

where $\|\cdot\|^{\prime}$ is the $l^{1}$-norm with respect to this basis. Since all norms on $V$ are equivalent, the result follows.

Coalgebra $Q S D E$ 's. Let $\varphi \in L(\mathcal{C} ; \mathcal{O}(\widehat{D}))$. Then $k \in \mathbb{P}\left(\mathcal{C}, \mathcal{E}_{D}\right)$ is a weak solution of the coalgebra quantum stochastic differential equation

$$
\mathrm{d} k_{t}=\mathrm{d} \Lambda_{\varphi}(t) \star k_{t}, \quad k_{0}=\iota \circ \epsilon
$$

( $\iota$ indicating an ampliation), if it satisfies

$$
\left\langle\varepsilon(f),\left(k_{t}(a)-\epsilon(a) I\right) \varepsilon(g)\right\rangle=\int_{0}^{t}\left\langle\hat{f}(s), \varphi\left(a_{(1)}\right) \hat{g}(s)\right\rangle\left\langle\varepsilon(f), k_{s}\left(a_{(2)}\right) \varepsilon(g)\right\rangle \mathrm{d} s .
$$

Thus a weak solution $k$ is necessarily weakly continuous and if $k \in \mathbb{P}_{2}\left(\mathcal{C}, \mathcal{E}_{D}\right)$ then

$$
k_{t}(a)=\epsilon(a) I+\int_{0}^{t} k_{s}\left(a_{(2)}\right) \mathrm{d} \Lambda_{\varphi\left(a_{(1)}\right)}(s)=\epsilon(a) I+\int_{0}^{t}\left(\varphi\left(a_{(1)}\right) \otimes k_{s}\left(a_{(2)}\right)\right) \mathrm{d} \Lambda_{s},
$$

where $\Lambda_{\varphi} \in \mathbb{P}_{\mathrm{Hc}}\left(\mathcal{C}, \mathcal{E}_{D}\right)$ is defined (in terms of creation, differential second quantisation and annihilation operators) as follows 


$$
\Lambda_{\varphi(a)}(t):=a^{*}\left(\varphi_{0}^{1}(a) \otimes\left|1_{[0, t[}\right\rangle\right)+\mathrm{d} \Gamma\left(\varphi_{1}^{1}(a) \otimes M_{[0, t[}\right)+a\left(\varphi_{1}^{0}(a) \otimes\left\langle 1_{[0, t[}\right|\right)+t \varphi_{0}^{0}(a) \otimes I,
$$

where $M_{[0, t[}$ denotes miltiplication by $1_{[0, t[}$ and so may be called a strong solution.

Remark. In view of (3.5) and (3.8) strong solutions necessarily belong to $\mathbb{P}_{\mathrm{Hc}}\left(\mathcal{C}, \mathcal{E}_{D}\right)$.

In terms of the map $\phi:=\left(\varphi \otimes \operatorname{id}_{\mathcal{C}}\right) \circ \Delta \in L(\mathcal{C} ; \mathcal{O}(\widehat{D}) \otimes \mathcal{C})$ a weak solution $k$ satisfies

$$
\left\langle\varepsilon(f),\left(k_{t}(a)-\epsilon(a) I\right) \varepsilon(g)\right\rangle=\int_{0}^{t}\left\langle\varepsilon(f), k_{s}\left(E^{\hat{f}(s)} \phi(a) E_{\hat{g}(s)}\right) \varepsilon(g)\right\rangle \mathrm{d} s,
$$

and a strong solution satisfies

$$
k_{t}(a)=\epsilon(a) I+\int_{0}^{t}\left(\hat{k}_{s} \circ \phi\right)(a) \mathrm{d} \Lambda_{s}
$$

where $\hat{k}_{s}:=\operatorname{id}_{\mathcal{O}(\widehat{D})} \otimes k_{s}$.

Proposition 4.2. The coalgebra QSDE (4.4) has at most one weak solution.

Proof. Let $k \in \mathbb{P}\left(\mathcal{C}, \mathcal{E}_{D}\right)$ be the difference of two weak solutions, and let $a \in \mathcal{C}, f, g \in \mathbb{S}_{D}$ and $T \geqslant 0$. Then $k \in \mathbb{P}_{\mathrm{wc}}\left(\mathcal{C}, \mathcal{E}_{D}\right) \subset \mathbb{P}_{\mathrm{wr}}\left(\mathcal{C}, \mathcal{E}_{D}\right)$, by (4.2). By iteration

$$
\left\langle\varepsilon(f), k_{t}(a) \varepsilon(g)\right\rangle=\int_{\Delta_{n}[0, t]}\left\langle\varepsilon(f), k_{s_{1}}\left(\phi_{\hat{g}\left(s_{1}\right)}^{\hat{f}\left(s_{1}\right)} \circ \cdots \circ \phi_{\hat{g}\left(s_{n}\right)}^{\hat{f}\left(s_{n}\right)}(a)\right) \varepsilon(g)\right\rangle \mathrm{d} \boldsymbol{s}
$$

for each $n \in \mathbb{N}$ and $t \in[0, T]$, where

$$
\phi_{\eta}^{\chi}:=E^{\chi} \phi(\cdot) E_{\eta}=\left(\left(\omega_{\chi, \eta} \circ \varphi\right) \otimes \operatorname{id}_{\mathcal{C}}\right) \circ \mathbf{\Lambda},
$$

and $\omega_{\chi, \eta}$ is the functional $R \mapsto\langle\chi, R \eta\rangle$ on $\mathcal{O}(\widehat{D})$. Since each $\phi_{\eta}^{\chi}$ leaves $\mathcal{C}_{a}$ invariant, fixing a norm for $\mathcal{C}_{a}$ and appealing to Lemma 4.1, we see that the integrand is bounded by

$$
\left(\max \left\{\left\|\phi_{\hat{g}(s)}^{\hat{f}(s)}\right\|: 0 \leqslant s \leqslant T\right\}\right)^{n} C_{f, g, T, \mathcal{C}_{a}},
$$

and so the result follows.

Next we establish an existence theorem for the coalgebra QSDE. Let $v^{\varphi}$ be the map $\mathcal{C} \rightarrow \varsigma_{D}$ defined by $v^{\varphi}(a)_{n}=v_{n}^{\varphi}(a)$ where $v_{0}^{\varphi}:=\epsilon$ and, in the notation (1.3),

$$
v_{n}^{\varphi}=\varphi^{\otimes n} \circ \mathbf{\Delta}_{n-1}: \mathcal{C} \rightarrow \mathcal{O}(\widehat{D})^{\otimes n} \subset \mathcal{O}\left(\widehat{D}^{\otimes n}\right) \text { for } n \geqslant 1 .
$$

Note the recursive identity

$$
v_{n+1}^{\varphi}=\left(\varphi \otimes v_{n}^{\varphi}\right) \circ \mathbf{\Lambda} .
$$

Note also that if $\mathcal{C}$ is involutive then

$$
\left(v^{\varphi}\right)^{\dagger}=v^{\psi}, \quad \text { where } \psi=\varphi^{\dagger} .
$$

In terms of the associated map $\phi:=\left(\varphi \otimes \operatorname{id}_{\mathcal{C}}\right) \circ \Delta$,

$$
v_{n}^{\varphi}=\epsilon_{n} \circ \phi^{(n)}
$$

where the maps $\phi^{(n)}: \mathcal{C} \rightarrow \mathcal{O}(\widehat{D})^{\otimes n} \otimes \mathcal{C}$ and $\epsilon_{n}: \mathcal{O}(\widehat{D})^{\otimes n} \otimes \mathcal{C} \rightarrow \mathcal{O}(\widehat{D})^{\otimes n}$ are defined (recursively) by 


$$
\phi^{(0)}=\mathrm{id}_{\mathcal{C}}, \epsilon_{0}=\epsilon \quad \text { and, } \quad \text { for } n \geqslant 1, \quad \phi^{(n)}=\left(\mathrm{id}_{n} \otimes \phi\right) \circ \phi^{(n-1)} \quad \text { and } \quad \epsilon_{n}=\mathrm{id}_{n} \otimes \epsilon,
$$

$\operatorname{id}_{n}$ standing for $\operatorname{id}_{\mathcal{O}(\widehat{D}) \otimes n}$.

Lemma 4.3. For any $\varphi \in L(\mathcal{C} ; \mathcal{O}(\widehat{D}))$, $v^{\varphi} \in L\left(\mathcal{C} ; \mathcal{H}_{D}\right)$. Moreover, if $\varphi$ is $\mathcal{O}^{\text {inv }}(\widehat{D})$-valued (respectively, $\mathcal{O}^{\dagger}(\widehat{D})$ valued, or $\mathcal{O}^{*}(\widehat{D})$-valued) then $v^{\varphi}$ is $\mathcal{H}_{D}^{\text {inv }}$-valued (respectively $\mathcal{H}_{D}^{\dagger}$-valued, or $\mathcal{H}_{D}^{*}$-valued).

Proof. Fix an element $a \in \mathcal{C} \backslash\{0\}$ and let $\mathcal{C}_{a}$ denote the coalgebra generated by $a$. By the Fundamental Theorem on coalgebras this is finite dimensional; let $a^{1}, \ldots, a^{N}$ be a basis in which $a^{1}=a$. Let $\left(v_{j k}^{i}\right)$ be the coefficients of $\boldsymbol{\Delta}$ (viewed as a map $\mathcal{C}_{a} \rightarrow \mathcal{C}_{a} \otimes \mathcal{C}_{a}$ ) with respect to this basis, and set

$$
T_{k}^{i}=\sum_{j} v_{j k}^{i} \varphi\left(a^{j}\right) \in \mathcal{O}(\widehat{D})
$$

Then $\phi\left(a^{i}\right)=\sum_{k} T_{k}^{i} \otimes a^{k}$ and

$$
v_{n}^{\varphi}(a)=\sum_{\mathbf{k}} \epsilon\left(a^{k_{n}}\right) T_{k_{1}}^{1} \otimes T_{k_{2}}^{k_{1}} \otimes \cdots \otimes T_{k_{n}}^{k_{n-1}},
$$

a sum of $N^{n}$ terms of the form $X_{1} \otimes \cdots \otimes X_{n}$ in which

$$
X_{i} \in\left\{T_{k}^{j}: 1 \leqslant j, k \leqslant N\right\} \cup\left\{\epsilon\left(a^{j}\right) T_{k}^{1}: 1 \leqslant j, k \leqslant N\right\},
$$

so $v^{\varphi}(a) \in \mathcal{H}_{D}$. Clearly $v^{\varphi}$ is linear. The rest is easily verified.

Theorem 4.4. Let $\varphi \in L(\mathcal{C} ; \mathcal{O}(\widehat{D}))$ and set $v=v^{\varphi}$. Then the process $k:=\Lambda \circ v$ strongly satisfies the coalgebra quantum stochastic differential equation (4.4).

Proof. By (3.12) and Lemma 4.3 the process $k$ is continuous. It therefore suffices to show that it satisfies the equation weakly. By (3.13), (3.14) and (4.8),

$$
\begin{aligned}
& \int_{0}^{t} \mathrm{~d} s\left\langle\hat{f}(s), \varphi\left(a_{(1)}\right) \hat{g}(s)\right\rangle\left\langle\varepsilon(f), k_{s}\left(a_{(2)}\right) \varepsilon(g)\right\rangle \\
& \quad=\int_{0}^{t} \mathrm{~d} s \int_{\Gamma_{[0, s]}} \mathrm{d} \tau\left\langle\pi_{\hat{f}}(\tau \cup s),\left(\varphi\left(a_{(1)}\right) \otimes v_{\# \tau}\left(a_{(2)}\right)\right) \pi_{\hat{g}}(\tau \cup s)\right\rangle\langle\varepsilon(f), \varepsilon(g)\rangle \\
& =\int_{\Gamma_{[0, t]}} \mathrm{d} \sigma\left(1-\delta_{\emptyset}(\sigma)\right)\left\langle\pi_{\hat{f}}(\sigma), v_{\# \sigma}(a) \pi_{\hat{g}}(\sigma)\right\rangle\langle\varepsilon(f), \varepsilon(g)\rangle \\
& =\left\langle\varepsilon(f), k_{t}(a) \varepsilon(g)\right\rangle-\epsilon(a)\langle\varepsilon(f), \varepsilon(g)\rangle,
\end{aligned}
$$

(for all $a \in \mathcal{C}, f, g \in \mathbb{S}_{D}$ and $t \geqslant 0$ ) and so, by (4.5), $k$ satisfies the equation weakly.

Thus the coalgebra QSDE (4.4) has a unique weak solution; it is a strong solution and is given by $\Lambda \circ v^{\varphi}$ — we denote it $l^{\varphi}$.

Lemma 4.5. Let $\varphi \in L(\mathcal{C} ; \mathcal{O}(\widehat{D}))$. Then $l^{\varphi} \in \mathbb{P}_{\mathrm{Hc}}\left(\mathcal{C}, \mathcal{E}_{D}\right)$ and the following holds.

(a) The map $\varphi \mapsto l^{\varphi}$ is injective.

(b) If $\varphi \in L\left(\mathcal{C} ; \mathcal{O}^{\dagger}(\widehat{D})\right)$ then $l^{\varphi} \in \mathbb{P}_{\mathrm{Hc}}^{\dagger}\left(\mathcal{C}, \mathcal{E}_{D}\right)$. 
(c) If $\mathcal{C}$ is unital then $l^{\varphi}$ is unital if and only if $\varphi(1)=0$.

(d) If $\mathcal{C}$ is involutive and $\varphi \in L\left(\mathcal{C} ; \mathcal{O}^{\dagger}(\widehat{D})\right)$ then $\left(l^{\varphi}\right)^{\dagger}=l^{\psi}$, where $\psi=\varphi^{\dagger}$. In particular, $l^{\varphi}$ is real if and only if $\varphi$ is real.

Proof. In view of (3.15), or the remark preceding Proposition 4.2, $l^{\varphi}$ belongs to $\mathbb{P}_{\mathrm{Hc}}\left(\mathcal{C}, \mathcal{E}_{D}\right)$.

(a) follows from the identity

$$
\langle\hat{c}, \varphi(a) \hat{d}\rangle=\lim _{t \rightarrow 0^{+}} t^{-1}\left(\left\langle\varepsilon\left(c_{[0, t[}\right), l_{t}^{\varphi}(a) \varepsilon\left(d_{[0, t[}\right)\right\rangle-\epsilon(a) \mathrm{e}^{t\langle c, d\rangle}\right),
$$

and the totality of $\{\hat{c}: c \in D\}$ in $\hat{\mathrm{k}}$.

(b) follows from Lemma 4.3 and (3.16).

(c) follows from (4.5), (4.10) and the unitality of $\epsilon$ and $\Delta$.

(d) By part (b) $l^{\varphi} \in \mathbb{P}_{\mathrm{Hc}}^{\dagger}\left(\mathcal{C}, \mathcal{E}_{D}\right)$ and by (4.9) and (3.16)

$$
\left(l^{\varphi}\right)^{\dagger}=\Lambda \circ\left(v^{\varphi}\right)^{\dagger}=\Lambda \circ v^{\psi}=l^{\psi} .
$$

The last part follows by injectivity.

Remark. If we cast $\varphi \in L(\mathcal{C} ; \mathcal{O}(\widehat{D}))$ in block matrix form:

$$
\varphi=\left[\begin{array}{cc}
\gamma & \alpha \\
\chi & v-\iota \circ \epsilon
\end{array}\right]
$$

where $\iota(z):=z I_{\mathrm{k}}$, then $\gamma \in L(\mathcal{C} ; \mathbb{C}), \chi \in L(\mathcal{C} ; L(\mathbb{C} ; D)), \alpha \in L(\mathcal{C} ; L(D ; \mathbb{C}))$ and $v \in L(\mathcal{C} ; \mathcal{O}(D))$, so that

$$
\varphi(a)=\left[\begin{array}{cc}
\gamma(a) & \alpha(a) \\
\chi(a) & v(a)-\epsilon(a) I
\end{array}\right]
$$

Moreover $\varphi \in L\left(\mathcal{C} ; \mathcal{O}^{\dagger}(\widehat{D})\right)$ if and only if $\alpha(\mathcal{C}) \subset\langle D|:=\{\langle d|: d \in D\}$ and $v(\mathcal{C}) \subset \mathcal{O}^{\dagger}(D)$. Thus if $\mathcal{C}$ is involutive then

$$
\varphi^{\dagger}=\left[\begin{array}{cc}
\gamma^{\dagger} & \chi^{\dagger} \\
\alpha^{\dagger} & v^{\dagger}-\iota \circ \epsilon
\end{array}\right]
$$

In particular,

$$
\varphi=\varphi^{\dagger} \quad \text { if and only if } \gamma=\gamma^{\dagger}, v=v^{\dagger} \text { and } \alpha=\chi^{\dagger} .
$$

\section{Quantum stochastic convolution cocycles}

In this section we first note that solutions of the coalgebra QSDE enjoy a cocycle relation. Heeding the fact that solutions are $\frac{1}{2}$-Hölder continuous we then establish the converse: every stochastic convolution cocycle in $\mathbb{P}_{\mathrm{Hc}}^{\dagger}\left(\mathcal{C}, \mathcal{E}_{D}\right)$ necessarily satisfies a coalgebra QSDE.

Lemma 5.1. Let $\varphi \in L(\mathcal{C} ; \mathcal{O}(\widehat{D}))$. Then $v:=v^{\varphi}$ (defined in (4.7)) satisfies

$$
v_{n+m}=\left(v_{n} \otimes v_{m}\right) \circ
$$

for all $n, m \in \mathbb{Z}_{+}$.

Proof. Since $v_{k}=\varphi^{\otimes k} \circ \boldsymbol{\Lambda}_{k-1}$, this reduces to the identity (1.4).

Recall the notational abuse (3.3). 
Proposition 5.2. Let $k=l^{\varphi}$ where $\varphi \in L(\mathcal{C} ; \mathcal{O}(\widehat{D}))$. Then $k$ satisfies the cocycle relation

$$
k_{s+t}=k_{s} \star\left(\sigma_{s} \circ k_{t}\right) .
$$

Proof. Set $v=v^{\varphi}$ and let $a \in \mathcal{C}$. In Sweedler's notation, the effect of the right-hand side on $a$ is $k_{s}\left(a_{(1)}\right) \otimes$ $\gamma_{s}\left(k_{t}\left(a_{(2)}\right)\right)=k_{s}\left(a_{(1)}\right) \sigma_{s}\left(k_{t}\left(a_{(2)}\right)\right)$ on $\mathcal{E}_{D,[0, s[} \otimes \mathcal{E}_{D,[s, \infty[}=\mathcal{E}_{D}$. Sandwiching with exponential vectors and applying (3.14) gives,

$$
\begin{aligned}
\langle\varepsilon( & \left.\left.f_{[0, s]}\right), k_{s}\left(a_{(1)}\right) \varepsilon\left(g_{[0, s[}\right)\right\rangle\left\langle\varepsilon\left(f_{[s, \infty[}\right), \sigma_{s}\left(k_{t}\left(a_{(2)}\right)\right) \varepsilon\left(g_{[s, \infty[}\right)\right\rangle \\
& =\int_{\Gamma_{[0, s[}} \mathrm{d} \sigma\left\langle\pi_{\hat{f}}(\sigma), v_{\# \sigma}\left(a_{(1)}\right) \pi_{\hat{g}}(\sigma)\right\rangle \int_{\Gamma_{[s, s+t[}} \mathrm{d} \tau\left\langle\pi_{\hat{f}}(\tau), v_{\# \tau}\left(a_{(2)}\right) \pi_{\hat{g}}(\tau)\right\rangle \mathrm{e}^{\langle f, g\rangle} \\
& =\int_{\Gamma_{[0, s+t[}} \mathrm{d} \omega\left\langle\pi_{\hat{f}}(\omega), v_{\# \omega \cap[0, s[}\left(a_{(1)}\right) \otimes v_{\# \omega \cap[s, s+t[}\left(a_{(2)}\right) \pi_{\hat{g}}(\omega)\right\rangle \mathrm{e}^{\langle f, g\rangle} .
\end{aligned}
$$

Applying Lemma 5.1, and identity (3.14) once more, we see that this is equal to $\left\langle\varepsilon(f), k_{s+t}(a) \varepsilon(g)\right\rangle$, as required.

Definition. A quantum stochastic convolution cocycle (on $\mathcal{C}$ with domain $\mathcal{E}_{D}$ ) is a process $k \in \mathbb{P}\left(\mathcal{C}, \mathcal{E}_{D}\right)$ satisfying (5.1), with initial condition $k_{0}=\iota \circ \epsilon: x \mapsto \epsilon(x) I$.

The collection of these is denoted $\mathbb{Q S C C}\left(\mathcal{C}, \mathcal{E}_{D}\right)$, and we adorn this notation with subscripts and superscript ${ }^{\dagger}$ according to the convention (4.1). Thus $\varphi \mapsto l^{\varphi}$ gives maps

$$
L(\mathcal{C} ; \mathcal{O}(\widehat{D})) \rightarrow \mathbb{Q S S C C}_{\mathrm{Hc}}\left(\mathcal{C}, \mathcal{E}_{D}\right) \quad \text { and } \quad L\left(\mathcal{C} ; \mathcal{O}^{\dagger}(\widehat{D})\right) \rightarrow \mathbb{Q S C C} \mathbb{C}_{\mathrm{Hc}}^{\dagger}\left(\mathcal{C}, \mathcal{E}_{D}\right)
$$

These maps are injective, by Lemma 4.5; our aim now is to establish bijectivity of the second map. When $k=l^{\varphi}$ we refer to $\varphi$ as the generator of the quantum stochastic convolution cocycle $k$. Note that if $k$ is a cocycle in $\mathbb{P}^{\dagger}\left(\mathcal{C}, \mathcal{E}_{D}\right)$, and $\mathcal{C}$ is involutive, then $k^{\dagger}$ is a cocycle too.

Lemma 5.3. Let $k \in \mathbb{Q S C C}\left(\mathcal{C}, \mathcal{E}_{D}\right)$. Then, for each $f, g \in \mathbb{S}_{D}$,

$$
\mathrm{e}^{-\left\langle f_{[0, t}, g_{[0, t]}\right\rangle}\left\langle\varepsilon\left(f_{[0, t]}\right), k_{t}(a) \varepsilon\left(g_{[0, t]}\right)\right\rangle=\prod_{i=0}^{n-1} \kappa_{t_{i+1}-t_{i}}^{f\left(t_{i}\right), g\left(t_{i}\right)}\left(a_{(i+1)}\right)
$$

where $0=t_{0} \leqslant t_{1} \leqslant \cdots \leqslant t_{n}=t$ contains the discontinuities of $f_{[0, t[}$ and $g_{[0, t[}, a_{(1)} \otimes \cdots \otimes a_{(n)}=\mathbf{\Delta}_{n-1}(a)$ (Sweedler-style), and

$$
\kappa_{t}^{c, d}:=\mathrm{e}^{-t\langle c, d\rangle}\left\langle\varepsilon\left(c_{[0, t[}\right), k_{t}(\cdot) \varepsilon\left(d_{[0, t[}\right)\right\rangle .
$$

Proof. This identity results from repeated application of the cocycle relation (5.1).

Corollary 5.4. Let $k \in \mathbb{Q S} \mathbb{C}_{\mathrm{wc}}\left(\mathcal{C}, \mathcal{E}_{D}\right)$. Then (5.3) defines continuous convolution semigroups of functionals on $\mathcal{C}$ : $\left(\kappa_{t}^{c, d}\right)_{t \geqslant 0}(c, d \in D)$.

Thus (5.2) is the analogue of the semigroup decomposition central to the analysis of Markovian cocycles in [27]. For a weakly continuous quantum stochastic convolution cocycle we shall refer to its associated CCSF's. Clearly two such cocycles with the same CCSF's must be equal.

Lemma 5.5. Let $k=l^{\varphi}$ for $\varphi \in L(\mathcal{C} ; \mathcal{O}(\widehat{D}))$. Then its associated CCSF's have generators $\{\langle\hat{c}, \varphi(\cdot) \hat{d}\rangle: c, d \in D\}$. 
Proof. Since $k_{t}(a)=\Lambda_{t}\left(v^{\varphi}(a)\right)$ this is an immediate consequence of (3.14) and the definition of $v^{\varphi}(4.7)$.

Proposition 5.6. Let $k \in \mathbb{Q S} \mathbb{C} \mathbb{C}_{\mathrm{c}}^{\dagger}\left(\mathcal{C}, \mathcal{E}_{D}\right)$. Define a map

$$
q: \widehat{D} \times \widehat{D} \rightarrow L(\mathcal{C} ; \mathbb{C}), \quad\left(\left(\begin{array}{l}
z \\
c
\end{array}\right),\left(\begin{array}{l}
w \\
d
\end{array}\right)\right) \mapsto[\overline{z-1} 1]\left[\begin{array}{ll}
\gamma_{0,0} & \gamma_{0, d} \\
\gamma_{c, 0} & \gamma_{c, d}
\end{array}\right]\left[\begin{array}{c}
w-1 \\
1
\end{array}\right],
$$

where $\left\{\gamma_{c, d}: c, d \in D\right\}$ are the generators of the CCSF's associated with $k$. Then $q$ is sesquilinear.

Proof. The proposition amounts to the sesquilinearity of each form $q_{a}:=q(\cdot, \cdot)(a)$. Thus let $a \in \mathcal{C}$. First note the identity

$$
q_{a}(\chi, \eta)=\lim _{t \rightarrow 0^{+}} t^{-1}\left\langle\xi(t),\left(w, d_{[0, t[},(2 !)^{-1 / 2}\left(d_{[0, t[}\right)^{\otimes 2}, \ldots\right)\right\rangle,
$$

for $\chi=\left(\begin{array}{l}z \\ c\end{array}\right)$ and $\eta=\left(\begin{array}{l}w \\ d\end{array}\right)$ in $\widehat{D}$, where

$$
\xi(t)=\left[k_{t}(a)^{\dagger}-\overline{\epsilon(a)} I\right]\left((z-1) \varepsilon(0)+\varepsilon\left(c_{[0, t[}\right)\right) .
$$

Thus if $\eta=\eta_{1}+\alpha \eta_{2}$ then

$$
q_{a}(\chi, \eta)-q_{a}\left(\chi, \eta_{1}\right)-\alpha q_{a}\left(\chi, \eta_{2}\right)=\lim _{t \rightarrow 0^{+}}\langle\xi(t), \eta(t)\rangle
$$

where

$$
\eta(t)=t^{-1}\left((n !)^{-1 / 2}\left[d^{\otimes n}-\left(d_{1}\right)^{\otimes n}-\alpha\left(d_{2}\right)^{\otimes n}\right] \otimes 1_{\left[0, t\left[^{n}\right.\right.}\right)_{n \geqslant 2} .
$$

Since $\eta$ is locally bounded and $\xi(t) \rightarrow 0$ as $t \rightarrow 0$, by the continuity of the process $\left(k_{t}(a)^{\dagger}\right)_{t \geqslant 0}$, this shows that $q_{a}$ is linear in its second argument. A very similar argument, this time using the continuity of the process $\left(k_{t}(a)\right)_{t \geqslant 0}$, shows that $q_{a}$ is conjugate linear in its first argument. The result follows.

Proposition 5.7. Let $k \in \mathbb{Q S C C} \mathbb{H c}_{\mathrm{Hc}}^{\dagger}\left(\mathcal{C}, \mathcal{E}_{D}\right)$ and let $q$ be defined as in Proposition 5.6. Then, for each a $\in \mathcal{C}$, the sesquilinear form $q(\cdot, \cdot)($ a) is separately continuous in each argument.

Proof. Let $\left\{\gamma_{c, d}: c, d \in D\right\}$ be the CCSF generators associated with $k$, and let $a \in \mathcal{C}$, and $\chi=\left(\begin{array}{l}z \\ c\end{array}\right), \eta=\left(\begin{array}{l}\omega \\ d\end{array}\right) \in \widehat{D}$. Then

$$
q(\chi, \eta)(a)=\bar{z}\left((w-1) \gamma_{0,0}(a)+\gamma_{0, d}(a)\right)+(w-1)\left(\gamma_{c, 0}(a)-\gamma_{0,0}(a)\right)+\left(\gamma_{c, d}(a)-\gamma_{0, d}(a)\right)
$$

and, for $e \in D$ and $T>0$,

$$
\begin{aligned}
\left|\gamma_{c, e}(a)-\gamma_{0, e}(a)\right| & =\lim _{t \rightarrow 0^{+}} t^{-1}\left|\left\langle\mathrm{e}^{-t\langle e, c\rangle} \varepsilon\left(c_{[0, t[}\right)-\varepsilon(0),\left(k_{t}(a)-\epsilon(a)\right) \varepsilon\left(e_{[0, t[}\right)\right\rangle\right| \\
& =\lim _{t \rightarrow 0^{+}} t^{-1}\left|\mathrm{e}^{-t\langle c, e\rangle}\left\langle\varepsilon\left(c_{[0, t[}\right)-\varepsilon(0),\left(k_{t}(a)-\epsilon(a)\right) \varepsilon\left(e_{[0, t[}\right)\right\rangle\right| \\
& \leqslant \limsup _{t \rightarrow 0^{+}} t^{-1 / 2}\left\|\varepsilon\left(c_{[0, t[}\right)-\varepsilon(0)\right\| t^{-1 / 2}\left\|\left[k_{t}(a)-\epsilon(a)\right] \varepsilon\left(e_{[0, T[}\right)\right\| \mathrm{e}^{-\|e\|^{2}(T-t) / 2} \\
& \leqslant\|c\| C(a, e, T)
\end{aligned}
$$

for some constant $C$ depending only on $a, e$ and $T$. Thus, setting $T=1$,

$$
|q(\chi, \eta)(a)| \leqslant|z|\left|(w-1) \gamma_{0,0}(a)+\gamma_{0, d}(a)\right|+\|c\|(|w-1| C(a, 0,1)+C(a, d, 1)) \leqslant M\|\chi\|
$$

for a constant $M$ depending only on $a$ and $\eta$. This establishes continuity in the first argument. Continuity in the second argument is proved by a very similar argument, this time using the Hölder-continuity of the process $\left(k_{t}(a)^{\dagger}\right)_{t} \geqslant 0$. 
Remark. Proposition 5.7 may be proved under the following weaker hypothesis: estimates

$$
\limsup _{t \rightarrow 0^{+}} t^{-1}\left|\left\langle\varepsilon\left(c_{[0, t[}\right)-\varepsilon(0), l_{t}(a) \varepsilon\left(d_{[0, t[}\right)\right\rangle\right| \leqslant M_{a, d}\|c\|,
$$

for constants $M_{a, d}$ depending only on $a$ and $d$, should be satisfied by both $k$ and $k^{\dagger}$.

Theorem 5.8. Let $k \in \mathbb{P}\left(\mathcal{C}, \mathcal{E}_{D}\right)$. Then the following are equivalent:

(i) $k \in \mathbb{Q S} \mathbb{C} \mathbb{C}_{\mathrm{Hc}}^{\dagger}\left(\mathcal{C}, \mathcal{E}_{D}\right)$.

(ii) $k=l^{\varphi}$ for some $\varphi \in L\left(\mathcal{C} ; \mathcal{O}^{\dagger}(\widehat{D})\right)$.

Proof. Let $k \in \mathbb{Q S C} \mathbb{C}_{\mathrm{Hc}}^{\dagger}\left(\mathcal{C}, \mathcal{E}_{D}\right)$ and let $\left\{\gamma_{c, d}: c, d \in D\right\}$ denote the CCSF generators associated with $k$. By Propositions 5.6 and 5.7, there is a map $\varphi \in L\left(\mathcal{C} ; \mathcal{O}^{\dagger}(\widehat{D})\right)$ such that

$$
\left\langle\left(\begin{array}{l}
z \\
c
\end{array}\right), \varphi(a)\left(\begin{array}{l}
w \\
d
\end{array}\right)\right\rangle=[\overline{z-1} 1]\left[\begin{array}{ll}
\gamma_{0,0}(a) & \gamma_{0, d}(a) \\
\gamma_{c, 0}(a) & \gamma_{c, d}(a)
\end{array}\right]\left[\begin{array}{c}
w-1 \\
1
\end{array}\right],
$$

in particular,

$$
\langle\hat{c}, \varphi(\cdot) \hat{d}\rangle=\gamma_{c, d} .
$$

Thus, by Lemma 5.5, the stochastic convolution cocycles $l^{\varphi}$ and $k$ have the same CCSF's and so coincide. Thus (i) implies (ii). The converse has already been established in Proposition 5.2 and Lemma 4.5(c).

Remark. The transformation between the family $\left\{\gamma_{c, d}: c, d \in D\right\}$ and $\varphi$ is a familiar one in the analysis of stochastic cocycle generators (cf. [27]).

As a special case of Theorem 5.8 , taking $\mathcal{C}=\mathbb{C}$, we obtain the following result.

Corollary 5.9. Let $X \in \mathbb{P}_{\mathrm{Hc}}^{\dagger}\left(\mathcal{E}_{D}\right)$ with $X_{0}=I$. Then the following are equivalent:

(i) $X$ is an operator Markovian cocycle: $X_{s+t}=X_{s} \sigma_{s}\left(X_{t}\right)$ for $s, t \geqslant 0$.

(ii) X satisfies a $Q S D E$ of the form $d X_{t}=\left(L \otimes X_{t}\right) d \Lambda_{t}$ for some $L \in \mathcal{O}^{\dagger}(\widehat{D})$.

This type of cocycle is used in the final section for perturbing quantum stochastic convolution cocycles on a general coalgebra.

\section{Multiplicativity}

So far our results have been for (involutive) coalgebras. For this section we fix a bialgebra $\mathcal{B}$. Since the quantum stochastic map $\Lambda$ is multiplicative $\mathcal{H}_{D}^{*} \rightarrow \mathbb{P}_{\mathrm{Hc}}^{\dagger}\left(\mathcal{E}_{D}\right)$ for the matrix-sum convolution product and pointwise weak operator product respectively (Theorem 3.3), we have reduced the question of multiplicativity for a stochastic convolution cocycle on $\mathcal{B}$ to that of the map $v^{\varphi}: \mathcal{B} \rightarrow \mathcal{H}_{D}^{*}$ derived from its generator $\varphi$ (in and above (4.7)).

For the proof of the following result we adopt some notations for converting a subset of the set $\{1, \ldots, n\}$ into subsets of $\{1, \ldots, n+1\}$ developed in [28]:

$$
\stackrel{\leftrightarrow}{v}:=\{1+k: k \in v\} \quad \text { and } \quad \vec{v}:=\{1\} \cup \stackrel{\sim}{v} .
$$

Proposition 6.1. Let $v=v^{\varphi}$ for $\varphi \in L\left(\mathcal{B} ; \mathcal{O}^{\text {inv }}(\widehat{D})\right)$. Then the following are equivalent: 
(i) $\varphi(a b)=\varphi(a) \epsilon(b)+\epsilon(a) \varphi(b)+\varphi(a) \Delta \varphi(b)$ for all $a, b \in \mathcal{B}$.

(ii) $v(a b)=v(a) * v(b)$ for all $a, b \in \mathcal{B}$.

Proof. (i) is contained in (ii) since $v_{1}(a b)=\varphi(a b)$ and $(v(a) * v(b))_{1}$ is the right-hand side of (i). Thus (ii) implies (i). Conversely, if (i) holds then $\mathcal{P}(1)$ holds where $\mathcal{P}(n)$ is the proposition

$$
\forall a, b \in \mathcal{C} \quad v_{n}(a b)=(v(a) * v(b))_{n} .
$$

By the multiplicativity of $\epsilon=v_{0}, \mathcal{P}(0)$ holds. Assume therefore that $\mathcal{P}(k)$ holds for $k \leqslant n$, and fix $a, b \in \mathcal{B}$. Employing Sweedler notation and using (4.8), $\mathcal{P}(1)$ and then $\mathcal{P}(n)$,

$$
\begin{aligned}
v_{n+1}(a b)= & \varphi\left(a_{(1)} b_{(1)}\right) \otimes v_{n}\left(a_{(2)} b_{(2)}\right) \\
= & {\left[\varphi\left(a_{(1)}\right) \epsilon\left(b_{(1)}\right)+\epsilon\left(a_{(1)}\right) \varphi\left(b_{(1)}\right)+\varphi\left(a_{(1)}\right) \Delta \varphi\left(b_{(1)}\right)\right] } \\
& \otimes \sum_{|\boldsymbol{\alpha}|=\{1, \ldots, n\}} v\left(a_{(2)}\right)\left(\alpha_{1} \cup \alpha_{2} ; n\right) \Delta\left[\alpha_{2} ; n\right] v\left(b_{(2)}\right)\left(\alpha_{2} \cup \alpha_{3} ; n\right),
\end{aligned}
$$

where the sum is over all partitions of the set $\{1, \ldots, n\}$ into three disjoint subsets. The identity

$$
\varphi\left(c_{(1)}\right) \otimes v\left(c_{(2)}\right)(\lambda ; n)=v(c)(\vec{\lambda} ; n+1),
$$

gives the following equalities

$$
\varphi\left(c_{(1)}\right) \otimes v\left(c_{(2)}\right)(\lambda ; n) \Delta[\lambda \cap \mu ; n] v(d)(\mu ; n)=v(c)(\vec{\lambda} ; n+1) \Delta[\stackrel{\circ}{v} ; n+1] v(d)(\vec{\mu} ; n+1),
$$

and

$$
\varphi\left(c_{(1)}\right) \Delta \varphi\left(d_{(1)}\right) \otimes v\left(c_{(2)}\right)(\lambda ; n) \Delta[\lambda \cap \mu ; n] v\left(d_{(2)}\right)(\mu ; n)=v(c)(\vec{\lambda} ; n+1) \Delta[\vec{v} ; n+1] v(d)(\vec{\mu} ; n+1),
$$

in which $v=\lambda \cap \mu$. Thus

$$
\begin{aligned}
& v_{n+1}(a b)=\sum_{|\boldsymbol{\alpha}|=\{1, \ldots, n\}}\left(v(a)\left(\overrightarrow{\alpha_{1}} \cup \stackrel{\overrightarrow{\alpha_{2}}}{ } ; n+1\right) \Delta\left[\overrightarrow{\alpha_{2}} ; n+1\right] v(b)\left(\overrightarrow{\alpha_{2}} \cup \stackrel{\overrightarrow{\alpha_{3}}}{\vec{\alpha}} ; n+1\right)\right.
\end{aligned}
$$

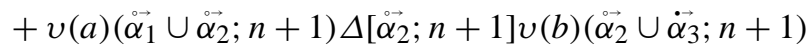

$$
\begin{aligned}
& \left.+v(a)\left(\overrightarrow{\alpha_{1}} \cup \overrightarrow{\alpha_{2}} ; n+1\right) \Delta\left[\overrightarrow{\alpha_{2}} ; n+1\right] v(b)\left(\overrightarrow{\alpha_{2}} \cup \stackrel{\alpha_{3}}{ } ; n+1\right)\right) \\
& =(v(a) * v(b))_{n+1} .
\end{aligned}
$$

The result therefore follows by induction.

Remark. The invariance assumption in the above proposition is sufficient for the application below. Replacing $\varphi(a) \Delta \varphi(b)$ by $\varphi(a) \cdot \Delta \varphi(b)$ and $v(a)$ by $v(a)^{\dagger *}$, the result holds under the alternative hypothesis $\varphi \in L\left(\mathcal{B} ; \mathcal{O}^{\dagger}(\widehat{D})\right)$.

Proposition 6.2. Let $\varphi \in L\left(\mathcal{B} ; \mathcal{O}^{*}(\widehat{D})\right)$ and set $k=l^{\varphi}$.

(a) If $k$ is weakly multiplicative then, for all $a, b \in \mathcal{B}$,

$$
\begin{aligned}
& \operatorname{Dom}\left(\varphi(a)^{\dagger}\right)^{*} \supset \operatorname{Ran} \Delta \varphi(b) \text { and } \\
& \varphi(a b)=\varphi(a) \epsilon(b)+\epsilon(a) \varphi(b)+\varphi(a) \cdot \Delta \varphi(b) .
\end{aligned}
$$

(b) Conversely, if $\varphi$ satisfies (6.1) then $k$ is weakly multiplicative. 
Proof. Let $v=v^{\varphi}$. If $k$ is weakly multiplicative then, using (4.10) and the Second Fundamental Formula,

$$
\langle\hat{c}, \varphi(a b) \hat{d}\rangle=\left\langle\varphi(a)^{\dagger} \hat{c}, \epsilon(b) \hat{d}\right\rangle+\left\langle\epsilon(a)^{*} \hat{c}, \varphi(b) \hat{d}\right\rangle+\left\langle\varphi(a)^{\dagger} \hat{c}, \Delta \varphi(b) \hat{d}\right\rangle
$$

for all $c, d \in D$. By sesquilinearity, this implies that

$$
\left\langle\varphi(a)^{\dagger} \chi, \Delta \varphi(b) \eta\right\rangle=\langle\chi,[\varphi(a b)-\varphi(a) \epsilon(b)-\epsilon(a) \varphi(b)] \eta\rangle
$$

for all $\chi, \eta \in \widehat{D}$, and so (a) holds.

Conversely, if $\varphi$ satisfies (6.1) then, by Lemma 4.4, $v$ is $\mathcal{H}_{D}^{*}$-valued and, by Proposition 6.1, $v(a) * v(b)=$ $v(a b)$. Thus, by Theorem 3.3, $k$ satisfies $\operatorname{Dom}\left(k_{t}(a)^{\dagger}\right)^{*} \supset \operatorname{Ran} k_{t}(b)$ and

$$
k_{t}(a) \cdot k_{t}(b)=\Lambda_{t}(v(a) * v(b))=\Lambda_{t}(v(a b))=k_{t}(a b),
$$

so $k$ is weakly multiplicative.

Remark. Further to the previous remark, this proposition also holds under the weaker hypothesis $\varphi \in L\left(\mathcal{B} ; \mathcal{O}^{\dagger}(\widehat{D})\right)$. The remark following Proposition 2.2 is relevant here; the proof of part (a) already only relies on $\varphi$ being $\mathcal{O}^{\dagger}(\widehat{D})$ valued.

In view of Theorem 3.3, the following characterisation is obtained from Lemma 4.5 and Proposition 6.2. Recall the algebra of processes defined in Theorem 3.3, and the remark on block matrix forms after Lemma 4.5.

Theorem 6.3. Let $k=l^{\varphi}$, where $\varphi \in L\left(\mathcal{B} ; \mathcal{O}^{*}(\widehat{D})\right)$, and suppose that $\mathcal{B}$ be a unital $*$-bialgebra. Then the following are equivalent:

(i) $k$ is unital and $*$-homomorphic as a map $\mathcal{B} \rightarrow(\mathcal{P}, \cdot)$.

(ii) $\varphi$ vanishes at $1_{\mathcal{B}}$ and satisfies

$$
\varphi\left(a^{*} b\right)=\varphi(a)^{*} \epsilon(b)+\epsilon(a)^{*} \varphi(b)+\varphi(a)^{*} \Delta \varphi(b) .
$$

(iii) $\varphi$ has block matrix form

$$
\left[\begin{array}{cc}
\gamma & \delta^{\dagger} \\
\delta & \rho-\iota \circ \epsilon
\end{array}\right]
$$

in which $\iota$ is the ampliation $z \mapsto z I_{\mathrm{k}}$;

$$
\rho: \mathcal{B} \rightarrow \mathcal{O}^{*}(D) \text { is a unital } * \text {-homomorphism; }
$$

$\delta: \mathcal{B} \rightarrow|D\rangle$ is a $\rho$ - $\epsilon$-derivation:

$$
\delta(a b)=\delta(a) \epsilon(b)+\rho(a) \delta(b) ;
$$

$\gamma: \mathcal{B} \rightarrow \mathbb{C}$ is linear and satisfies

$$
\gamma\left(a^{*} b\right)=\gamma(a)^{*} \epsilon(b)+\epsilon(a)^{*} \gamma(b)+\delta(a)^{*} \delta(b) .
$$

Following Paul-André Meyer [31] we shall refer to such $(\gamma, \delta, \rho)$ as a $D$-Schürmann triple on $\mathcal{B}$.

\section{Quantum Lévy processes}

In this section we describe Schürmann's theorem on the reconstruction of a quantum Lévy process from its 'generator', and give a new simple proof of its realisation as a process on Fock space.

For this section let $\mathcal{B}$ be a unital $*$-bialgebra. By a quantum Lévy process on $\mathcal{B}$ over a unital $*$-algebra-with-state $(\mathcal{A}, \omega)$ is meant a family $\left\{j_{s, t}: \mathcal{B} \rightarrow \mathcal{A} \mid 0 \leqslant s \leqslant t\right\}$ of unital $*$-homomorphisms satisfying 
(QL1) $j_{r, t}=j_{r, s} \star j_{s, t}$ for $0 \leqslant r \leqslant s \leqslant t ; j_{t, t}(x)=\epsilon(x) 1_{\mathcal{A}}$ for $t \geqslant 0$;

(QL2) $\left\{j_{s_{i}, t_{i}}(\mathcal{B}): i=1, \ldots n\right\}$ commute, and

$$
\omega\left(\prod_{i=1}^{n} j_{s_{i}, t_{i}}\left(x_{i}\right)\right)=\prod_{i=1}^{n} \omega\left(j_{s_{t}, t_{i}}\left(x_{i}\right)\right)
$$

whenever the intervals $\left[s_{1}, t_{1}\left[, \ldots,\left[s_{n}, t_{n}[\right.\right.\right.$ are disjoint;

(QL3) $\omega \circ j_{s, t}=\omega \circ j_{0, t-s}$ for $0 \leqslant s \leqslant t$;

(QL4) $\omega \circ j_{0, t}(x) \rightarrow \epsilon(x)$ as $t \rightarrow 0$.

(QL1) is known as the increment property [36,12]; the others respectively as (tensor) independence of increments, time-homogeneity and continuity. It is immediately verified that

$$
\kappa_{t}:=\omega \circ j_{0, t}
$$

defines a continuous convolution semigroup of states on $\mathcal{B}$, called the one-dimensional distribution of the quantum Lévy process; its generator is also referred to as the generator of the quantum Lévy process.

Quantum Lévy processes $j^{i}$ on $\mathcal{B}$ over $\left(\mathcal{A}^{i}, \omega^{i}\right)(i=1,2)$ are said to be equivalent if they satisfy

$$
\omega^{1}\left(\prod_{i=1}^{n} j_{s_{i}, t_{i}}^{1}\left(x_{i}\right)\right)=\omega^{2}\left(\prod_{i=1}^{n} j_{s_{i}, t_{i}}^{2}\left(x_{i}\right)\right)
$$

for all $n \in \mathbb{N}$, intervals $\left[s_{i}, t_{i}\right.$ [ and elements $x_{i}(i=1, \ldots, n)$. In view of the axioms (QL1-3) it is clear that two quantum Lévy processes are equivalent if and only if their one-dimensional distributions coincide - equivalently, if their generators are equal.

Let $k \in \mathbb{Q S C} \mathbb{C}_{\mathrm{Hc}}^{\dagger}\left(\mathcal{B}, \mathcal{E}_{D}\right)$ be unital, real and weakly multiplicative with generator $\varphi$. Then, setting

$$
\begin{aligned}
& \mathcal{A}^{\varphi}=\operatorname{Lin}\left\{k_{s_{1}}\left(x_{1}\right) \cdot \sigma_{s_{1}}\left(k_{s_{2}-s_{1}}\left(x_{2}\right)\right) \cdots \cdot \sigma_{s_{n-1}}\left(k_{s_{n}-s_{n-1}}\left(x_{n}\right)\right): n \in \mathbb{N}, 0 \leqslant s_{1} \leqslant \cdots \leqslant s_{n}, x_{1}, \ldots, x_{n} \in \mathcal{B}\right\}, \\
& j_{s, t}^{\varphi}=\sigma_{s} \circ k_{t-s}: \mathcal{B} \rightarrow \mathcal{A}^{\varphi}, \text { and } \\
& \omega^{\varphi}=\omega_{\Omega} \mid \mathcal{A}^{\varphi},
\end{aligned}
$$

$\mathcal{A}^{\varphi}$ is a unital *-algebra in the involutive linear space $\mathcal{O}^{\dagger}\left(\mathcal{E}_{D}\right)$ with product given by $(1.10), \omega^{\varphi}$ is a state on $\mathcal{A}^{\varphi}$ and it is easily checked that $j^{\varphi}$ is a quantum Lévy process over $\left(\mathcal{A}^{\varphi}, \omega^{\varphi}\right)$ with generator $\gamma$, where $\gamma=\varphi_{0}^{0}$ (the top-left component of the block matrix form of $\varphi$ ). Let us call this type a Fock space quantum Lévy process.

Note that since a quantum Lévy process is unital (real) and positive, its generator vanishes on $1_{\mathcal{B}}$, is real and is conditionally positive (see (1.8)).

Theorem 7.1 [35,36]. Let $\gamma$ be a real, conditionally positive linear functional on $\mathcal{B}$ vanishing at $1_{\mathcal{B}}$. Then there is a Fock space quantum Lévy process with generator $\gamma$.

Proof. GNS-style, let $D=\operatorname{Ker} \epsilon / N$ where

$$
N=\left\{a \in \operatorname{Ker} \epsilon \mid \gamma\left(a^{*} a\right)=0\right\} .
$$

Then $([a],[b]) \mapsto \gamma\left(a^{*} b\right)$ defines an inner product on $D$; let $\mathrm{k}$ be its completion. Then $\rho(a):[c] \mapsto[a c]$ defines operators on $D$ which make up a unital representation of $\mathcal{B}$ on $D$ satisfying

$$
\langle\rho(a)[b],[c]\rangle=\left\langle[b], \rho\left(a^{*}\right)[c]\right\rangle .
$$

Thus $\rho$ is a unital $*$-homomorphism $\mathcal{B} \rightarrow \mathcal{O}^{*}(D)$. Moreover the linear map $\delta: a \mapsto|d(a)\rangle$, where $d(a)=[a-$ $\epsilon(a) I]$, is easily seen to be a $\rho-\epsilon$-derivation $\mathcal{B} \rightarrow|D\rangle$ satisfying

$$
\delta(a)^{*} \delta(b)=\gamma\left(a^{*} b\right)-\gamma(a)^{*} \epsilon(b)-\epsilon(a)^{*} \gamma(b) .
$$


Set $k=l^{\varphi}$, where $\varphi$ is the map $\mathcal{B} \rightarrow \mathcal{O}^{*}(\widehat{D})$ with block matrix form given by the prescription (6.2). Then Theorem 6.3 implies that $k$ is $*$-homomorphic (i.e. real and weakly multiplicative) and unital. Since $\varphi_{0}^{0}=\gamma$ the result follows.

Corollary 7.2. Every quantum Lévy process is equivalent to a Fock space quantum Lévy process.

\section{Perturbation}

Consider the case of the trivial bialgebra $\mathbb{C}$, and let $\varphi \in L(\mathbb{C} ; \mathcal{O}(\widehat{D}))$. Then $\varphi$ and $l^{\varphi}$ are determined by the operator $L:=\varphi(1) \in \mathcal{O}(\widehat{D})$ and the process $X^{L}:=l^{\varphi}(1) \in \mathbb{P}_{\mathrm{Hc}}\left(\mathcal{E}_{D}\right)$ which satisfies the operator QSDE

$$
\mathrm{d} X_{t}=\left(L \otimes X_{t}\right) \mathrm{d} \Lambda_{t}, \quad X_{0}=I .
$$

These processes have explicit action on exponential vectors:

$$
X_{t}^{L} \varepsilon(f)=\exp \left(t z+\int_{0}^{t} \beta(f(s)) \mathrm{d} s\right) \varepsilon\left((R f)_{[0, t[}+d_{[0, t[}\right)
$$

where

$$
\left[\begin{array}{cc}
z & \beta \\
|d\rangle & R-I
\end{array}\right], \quad \text { with } z \in \mathbb{C}, \mathrm{d} \in \mathrm{k}, \beta \in L(D ; \mathbb{C}), \text { and } R \in \mathcal{O}(D),
$$

is the block matrix form of $L$. From either of the above descriptions it is clear that the map $L \mapsto X^{L}$ is injective $\mathcal{O}(\widehat{D}) \rightarrow \mathbb{P}_{\mathrm{Hc}}\left(\mathcal{E}_{D}\right)$. Moreover if $L \in \mathcal{O}^{\dagger}(\widehat{D})$ (equivalently, $R \in \mathcal{O}^{\dagger}(D)$ and $\beta=\langle c|$ for some $c \in \mathrm{k}$ ) then $X^{L} \in$ $\mathbb{P}_{\mathrm{Hc}}^{\dagger}\left(\mathcal{E}_{D}\right)$ and $\left(X^{L}\right)^{\dagger}=X^{M}$ where $M=L^{\dagger}$. Similarly, if $L \in \mathcal{O}^{\text {inv }}(\widehat{D})$ (equivalently, $R \in \mathcal{O}^{\text {inv }}(D)$ and $d \in D$ ) then $X_{t}^{L} \in \mathcal{O}^{\text {inv }}\left(\mathcal{E}_{D}\right)$ for each $t$. If $L \in \mathcal{O}(\widehat{D})$ and $M \in \mathcal{O}^{\text {inv }}(\widehat{D})$ then, by the explicit action (8.2),

$$
X^{L} X^{M}=X^{L} M
$$

where

$$
L \triangleleft M:=L+M+L \Delta M
$$

By the above injectivity $\left(\mathcal{O}^{\text {inv }}(\widehat{D}), \diamond\right)$ is a semigroup with identity 0 ; clearly $\left(\mathcal{O}^{*}(\widehat{D}), \diamond\right)$ is an involutive semigroup: $(L \backslash M)^{\dagger}=M^{\dagger} L^{\dagger}$. Note that these identities contain the Weyl commutation relations.

The above formula implies that for $L \in \mathcal{O}^{*}(\widehat{D})$

$$
X^{L} \text { is isometric } \Longleftrightarrow L^{\dagger} \downarrow L=0 \text {, whereas } X^{L} \text { is coisometric } \Longleftrightarrow L L^{\dagger}=0,
$$

cf. analogous characterisations described in [26].

In the next proposition, (8.4) is extended by left and right actions of (parts of) $\mathcal{O}^{*}(\widehat{D})$ on $L(\mathcal{C} ; \mathcal{O}(\widehat{D}))$, for a coalgebra $\mathcal{C}$.

Proposition 8.1. Let $\varphi \in L(\mathcal{C} ; \mathcal{O}(\widehat{D}))$ and let $L, M \in \mathcal{O}(\widehat{D})$.

(a) If $\varphi \in L\left(\mathcal{C} ; \mathcal{O}^{\dagger}(\widehat{D})\right)$ and $M \in \mathcal{O}^{\text {inv }}(\widehat{D})$ then

$$
l^{\varphi}(\cdot) X^{M}=l^{\varphi} M
$$

(b) If $L \in \mathcal{O}^{\dagger}(\widehat{D})$ and $\varphi \in L\left(\mathcal{C} ; \mathcal{O}^{\text {inv }}(\widehat{D})\right)$ then

$$
l_{t}^{\varphi}(\mathcal{C}) \subset \operatorname{Dom}\left(X_{t}^{L}\right)^{\dagger *} \text { and } X^{L} \cdot l^{\varphi}(\cdot)=l^{L} \varphi,
$$


where

$$
(\varphi M)(a):=\varphi(a)(I+\Delta M)+\epsilon(a) M
$$

and

$$
(L \vee \varphi)(a):=(I+L \Delta) \varphi(a)+\epsilon(a) L .
$$

Proof. These follow easily from the two Fundamental Formulae.

That these extend (8.4) is confirmed by setting $\mathcal{C}=\mathbb{C}$ and $a=1$.

Let $\varphi \in L\left(\mathcal{C} ; \mathcal{O}^{*}(\widehat{D})\right)$ and $L_{i} \in \mathcal{O}^{*}(\widehat{D})(i=1,2)$. Then their block matrix forms (see (4.11)) are respectively

$$
\left[\begin{array}{cc}
\gamma & \alpha \\
\chi & v-\iota \circ \epsilon
\end{array}\right] \text { and }\left[\begin{array}{cc}
z_{i} & \left\langle c_{i}\right| \\
\left|d_{i}\right\rangle & R_{i}-I
\end{array}\right] \text {, }
$$

where $z_{i} \in \mathbb{C}, c_{i} \in D, R_{i} \in \mathcal{O}^{*}(D)$, and

$$
\begin{aligned}
\left(L_{1}^{\dagger} \varphi L_{2}\right)(a) & =\left(I+\Delta L_{1}\right)^{\dagger} \varphi(a)\left(I+\Delta L_{2}\right)+\epsilon(a) L_{1}^{\dagger} L_{2} \\
& =\left[\begin{array}{cc}
\tilde{\gamma}(a) & \left(\alpha(a)+\left\langle d_{1}\right| v(a)\right) R_{2}+\epsilon(a)\left\langle c_{2}\right| \\
R_{1}^{\dagger}\left(\chi(a)+v(a)\left|d_{2}\right\rangle\right)+\epsilon(a)\left|c_{1}\right\rangle & R_{1}^{\dagger} v(a) R_{2}-\epsilon(a) I
\end{array}\right]
\end{aligned}
$$

where

$$
\tilde{\gamma}(a)=\gamma(a)+\left(z_{1}^{*}+z_{2}\right) \epsilon(a)+\alpha(a)\left|d_{2}\right\rangle+\left\langle d_{1}\right| \chi(a)+\left\langle d_{1}, v(a) d_{2}\right\rangle .
$$

In particular, consider conjugation by a single map $L \in \mathcal{O}^{*}(\widehat{D})$ :

$$
\tilde{\varphi}=L^{\dagger} \diamond \varphi L
$$

where $\varphi \in L\left(\mathcal{B} ; \mathcal{O}^{*}(\widehat{D})\right)$ for a unital $*$-bialgebra $\mathcal{B}$. It is easily checked that if $l^{\varphi}$ is real then $l^{\tilde{\varphi}}$ is real; if $l^{\varphi}$ is unital then

$$
l^{\tilde{\varphi}} \text { is unital } \Longleftrightarrow X^{L} \text { is isometric; }
$$

and if $l^{\varphi}$ is weakly multiplicative then $l^{\tilde{\varphi}}$ is weakly multiplicative if and only if

$$
\forall a \in \mathcal{B} \quad(\Delta L+I)^{*}(\Delta \varphi(a)+\epsilon(a) I)^{\dagger} L L^{\dagger}(\Delta \varphi(a)+\epsilon(a) I)(\Delta L+I)=0 .
$$

Therefore, considering perturbations by unitary (Weyl) cocycles, we obtain the action of the Euclidean group of $D$ on Schürmann triples associated with unital *-homomorphic quantum stochastic convolution cocycles (cf. [11]). This action has a simple matricial description, namely if

$$
L=\left[\begin{array}{cc}
\mathrm{i} \mu-\frac{1}{2}\|v\|^{2} & -\langle v| V \\
|v\rangle & V-I
\end{array}\right]
$$

where $\mu \in \mathbb{R}, v \in D$ and $V \in \mathcal{O}^{*}(D)$ is unitary, then

$$
\tilde{\varphi}(a)=\left[\begin{array}{cc}
1 & \langle v| \\
0 & V^{*}
\end{array}\right] \varphi(a)\left[\begin{array}{cc}
1 & 0 \\
|v\rangle & V
\end{array}\right] .
$$

Thus if

$$
\varphi=\left[\begin{array}{cc}
\gamma & \delta^{\dagger} \\
\delta & \rho-\iota \circ \epsilon
\end{array}\right]
$$

then 


$$
\begin{aligned}
& \tilde{\gamma}(a)=\gamma(a)+\delta^{\dagger}(a)|v\rangle+\langle v| \delta(a)+\langle v,(\rho(a)-\epsilon(a) I) v\rangle, \\
& \tilde{\delta}(a)=V^{*}(\delta(a)+(\rho(a)-\epsilon(a) I)|v\rangle) \text { and } \\
& \tilde{\rho}(a)=V^{*} \rho(a) V .
\end{aligned}
$$

Notice that the part of the action determined by $V$ is trivial in the sense that only a unitary transformation of the Schürmann triple $(\lambda, \delta, \rho)$ leaving $\lambda$ invariant is effected, so that the perturbed quantum Lévy process $l^{\tilde{\varphi}}$ is equivalent to the unperturbed one $l^{\varphi}$. For nonzero $v$ the perturbation still does not change the characteristics of the quantum Lévy process. Thus Gaussian processes remain Gaussian and the same is true for Poisson and drift processes (see [11]).

\section{Note added in proof}

The principal results of this paper have now been extended to a topological context (see [25]).

\section{Acknowledgements}

This work was carried out at the University of Nottingham where AGS was supported first by the EU Research and Training Network Quantum Probability with Applications to Physics, Information Theory and Biology, and later by a UK an Overseas Research Scholarship. AGS also acknowledges support from KBN Research Grant 2P03A 03024. We are very grateful to Uwe Franz and Michael Schürmann, the organisers of the 2003 Spring School in Greifswald: Quantum Independent Increment Processes, after which our investigating this topic was (almost) inevitable.

\section{References}

[1] L. Accardi, On the quantum Feynman-Kac formula, Rend. Sem. Mat. Fis. Milano 48 (1978) 135-180.

[2] L. Accardi, J.-L. Journé, J.M. Lindsay, On multidimensional Markovian cocycles, in: L. Accardi, W. von Waldenfels (Eds.), Quantum Probability and Applications IV, Lecture Notes in Math., vol. 1396, Springer-Verlag, Berlin, 1989, pp. 59-67.

[3] L. Accardi, A. Mohari, On the structure of classical and quantum flows, J. Funct. Anal. 135 (2) (1996) $421-455$.

[4] W. Arveson, Noncommutative Dynamics and E-Semigroups, Springer-Verlag, New York, 2003.

[5] L. Accardi, M. Schürmann, W. von Waldenfels, Quantum independent increment processes on superalgebras, Math. Z. 198 (4) (1988) 451-477.

[6] D. Applebaum, B.V.R. Bhat, J. Kustermans, J.M. Lindsay, Quantum Independent Increment Processes I: From Classical Probability to Quantum Stochastics, U. Franz, M. Schürmann (Eds.), Lecture Notes in Math., vol. 1865, Springer-Verlag, Heidelberg, 2005.

[7] O.E. Barndorff-Nielsen, U. Franz, G. Gohm, B. Krümmerer, S. Thorbjørsen, Quantum Independent Increment Processes II: Structure of Quantum Lévy Processes, Classical Probability and Physics, U. Franz, M. Schürmann (Eds.), Lecture Notes in Math., vol. 1866, SpringerVerlag, Heidelberg, in press.

[8] W.S. Bradshaw, Stochastic cocycles as a characterisation of quantum flows, Bull. Sci. Math. 116 (1) (1992) 1-34.

[9] M.P. Evans, R.L. Hudson, Perturbations of quantum diffusions, J. London Math. Soc. (2) 41 (2) (1990) 373-384.

[10] F. Fagnola, Characterization of isometric and unitary weakly differentiable cocycles in Fock space, in: L. Accardi (Ed.), Quantum Probability and Related Topics VIII, World Scientific, Singapore, 1993, pp. 143-164.

[11] U. Franz, Lévy processes on quantum groups and dual groups, in [7].

[12] U. Franz, R. Schott, Stochastic Processes and Operator Calculus on Quantum Groups, Math. Appl., vol. 490, Kluwer Academic, Dordrecht, 1999.

[13] P. Glockner, Quantum stochastic differential equations on *-bialgebras, Math. Proc. Cambridge Philos. Soc. 109 (3) (1991) $571-595$.

[14] D. Goswami, J.M. Lindsay, S.J. Wills, A stochastic Stinespring theorem, Math. Ann. 319 (4) (2001) 647-673.

[15] A. Guichardet, Symmetric Hilbert Spaces and Related Topics, Lecture Notes in Math., vol. 267, Springer, Heidelberg, 1970.

[16] J. Hellmich, C. Köstler, B. Kümmerer, Noncommutative continuous Bernoulli shifts, Preprint, Queen's University, Kingston, 2004.

[17] R.L. Hudson, Unitarity and multiplicativity via higher Itô product formula, Tatra Mt. Math. Publ. 10 (1997) 95-108. 
[18] R.L. Hudson, J.M. Lindsay, On characterizing quantum stochastic evolutions, Math. Proc. Cambridge Philos. Soc. 102 (2) (1987) $363-369$.

[19] R.L. Hudson, K.R. Parthasarathy, Quantum Itô's formula and stochastic evolutions, Comm. Math. Phys. 93 (3) (1984) $301-323$.

[20] J.-L. Journé, Structure des cocycles markoviens sur l'espace de Fock, Probab. Theory Related Fields 75 (2) (1987) 291-316.

[21] J. Kustermans, Locally compact quantum groups, in [6].

[22] J. Kustermans, S. Vaes, Locally compact quantum groups, Ann. Sci. École Norm. Sup. (4) 33 (6) (2000) 837-934.

[23] J.M. Lindsay, Integral-sum kernel operators, in: S. Attal, J.M. Lindsay (Eds.), Quantum Probability Communications XII, World Scientific, Singapore, 2003, pp. 1-21.

[24] J.M. Lindsay, Quantum stochastic analysis — an introduction, in [6].

[25] J.M. Lindsay, A.G. Skalski, Quantum stochastic convolution cocycles-algebraic and $C^{*}$-algebraic, in: M. Bożejko, R. Lenczewski, W. Młotkowski, J. Wysoczański (Eds.), Quantum Probability and Related Topics, Banach Center Publications, Polish Academy of Sciences, Warsaw, 2005, in press.

[26] J.M. Lindsay, S.J. Wills, Existence, positivity, and contractivity for quantum stochastic flows with infinite dimensional noise, Probab. Theory Related Fields 116 (4) (2000) 505-543.

[27] J.M. Lindsay, S.J. Wills, Markovian cocycles on operator algebras, adapted to a Fock filtration, J. Funct. Anal. 178 (2) (2000) $269-305$.

[28] J.M. Lindsay, S.J. Wills, Homomorphic Feller cocycles on a $C^{*}$-algebra, J. London Math. Soc. (2) 68 (1) (2003) $255-272$.

[29] J.M. Lindsay, S.J. Wills, Operator Markovian cocycles via associated semigroups, Preprint, 2004.

[30] H. Maassen, Quantum Markov processes on Fock space described by integral kernels, in: L. Accardi, W. von Waldenfels (Eds.), Quantum Probability and Applications II, Lecture Notes in Math., vol. 1136, Springer-Verlag, Berlin, 1985, pp. 361-374.

[31] P.-A. Meyer, Quantum Probability for Probabilists, second ed., Lecture Notes in Math., vol. 1538, Springer-Verlag, Berlin, 1995.

[32] K.R. Parthasarathy, Introduction to Quantum Stochastic Calculus, Birkhäuser, Basel, 1992.

[33] K. Schmüdgen, Unbounded Operator Algebras and Representation Theory, Akademie-Verlag, Berlin, 1990.

[34] M. Schürmann, Noncommutative stochastic processes with independent and stationary increments satisfy quantum stochastic differential equations, Probab. Theory Related Fields 84 (4) (1990) 473-490.

[35] M. Schürmann, White noise on involutive bialgebras, in: Quantum Probability \& Related Topics VI, World Scientific, Singapore, 1991, pp. 401-419.

[36] M. Schürmann, White Noise on Bialgebras, Lecture Notes in Math., vol. 1544, Springer, Heidelberg, 1993.

[37] M. Schürmann, Operator processes majorizing their quadratic variation, Infin. Dimens. Anal. Quantum Probab. Relat. Top. 3 (1) (2000) 99-120.

[38] A.V. Skorohod, Operator stochastic differential equations and stochastic semigroups, Uspekhi Mat. Nauk 37 (6) (1982) 157-183, (228) (in Russian); Russian Math. Surveys 37 (6) (1982) 177-204.

[39] M.E. Sweedler, Hopf Algebras, Benjamin, New York, 1969.

[40] S.L. Woronowicz, Compact matrix pseudogroups, Comm. Math. Phys. 111 (4) (1987) 613-665.

[41] S.L. Woronowicz, Compact quantum groups, in: A. Connes, K. Gawedzki, J. Zinn-Justin (Eds.), Symétries Quantiques, Proceedings, Les Houches, 1995, North-Holland, Amsterdam, 1998, pp. 845-884. 